\title{
Nature of Charge Carriers in Long Doped Oligothiophenes: The Effect of Counterions
}

\author{
Natalia Zamoshchik, ${ }^{\dagger}$ Ulrike Salzner, $*, \star$ and Michael Bendikov*, ${ }^{*}$ \\ Department of Organic Chemistry, Weizmann Institute of Science, 76100 Rehovot, Israel, and Department of \\ Chemistry, Bilkent University, 06800 Bilkent, Ankara, Turkey
}

Received: November 24, 2007; Revised Manuscript Received: March 7, 2008

\begin{abstract}
A series of oligothiophene dications doped with $\mathrm{Cl}_{3}{ }^{-}$ions were studied using density functional theory (DFT) at the B3LYP/6-31G(d) level. The balance between the bipolaron and polaron pair states was addressed by studying the closed-shell singlet, open-shell singlet, and triplet states of oligothiophene divalent salts using the relative energies of the different isomers, differences between the singlet and triplet states, bond length alternation analysis, charge distribution analysis, and isodesmic reactions. We found that contribution of polaron pair state to the electronic structure of the oligothiophene divalent salts is not observed in $8 \mathrm{~T}^{2+}\left(\mathrm{Cl}_{3}{ }^{-}\right)_{2}$ (short oligothiophene salts), appears in $12 \mathrm{~T}^{2+}\left(\mathrm{Cl}_{3}^{-}\right)_{2}$ (medium-sized oligothiophene salts), and becomes the dominant in $20 \mathrm{~T}^{2+}\left(\mathrm{Cl}_{3}{ }^{-}\right)_{2}$ (long oligothiophene salts). Bipolarons are intrinsically unstable with respect to dissociation into polaron pairs regardless of the presence of counterions. Thus, even in the presence of counterions, we did not observe any bipolaron stabilization energy, however, doping ions localize polarons. The singlet and triplet states are energetically degenerate for long oligothiophene divalent salts, such as $20 \mathrm{~T}^{2+}\left(\mathrm{Cl}_{3}{ }^{-}\right)_{2}$.
\end{abstract}

\section{Introduction}

Oligothiophenes (nT) and polythiophene are among the most promising and best studied organic electronic materials. ${ }^{1}$ Oligoand polythiophenes are widely used in applications such as fieldeffect transistors (FET), ${ }^{2,3}$ organic light-emitting diodes (OLED), ${ }^{4,5}$ solar cells, ${ }^{6,7}$ etc, and polythiophenes have significant potential as electrochromic devices. ${ }^{8}$ One of the main advantages of these materials is that their conductivity spans the range from semiconductor to conductor and can be easily and reversibly controlled by doping.

A major challenge in the field of organic electronic materials is to understand the basic mechanisms involved in charge transport. One of the most fundamental questions in understanding the conductivity of doped polythiophenes is the nature of the charge carriers, that is, whether they are bipolarons (dications) localized over a limited section of the chain or polaron pairs (pairs of radical cations) separated by nearly undistorted sections of the chain. ${ }^{9-12}$ Such understanding is crucial to the design of new materials with improved properties. The relative stability of polaron pairs and bipolarons in conjugated oligomers and polymers is still an open question that is difficult to address experimentally. ${ }^{11-14}$ Different theoretical and experimental studies arrived at opposite conclusions. Some experimental studies ${ }^{13}$ contended that bipolarons are the major charge carriers in polythiophene at doping levels as low as $\sim 1 \%$ and that polarons are preferred only at very low doping levels $(<0.1 \%)$. Nevertheless, some recent studies suggested that mostly polarons are formed in doped polythiophenes and in long oligothiophenes. ${ }^{11,14}$

It is also difficult to solve the problem theoretically, as different levels of theory lead to contradictory predictions. Early theoretical studies based on the Hückel method ${ }^{15}$ suggested that bipolarons are intrinsically stable in long conjugated chains with bipolaron binding energies of between about quater to half $\mathrm{eV}$. Geometries used in the band structure calculations of the

* To whom correspondence should be addressed. E-mail: (M.B.) michael.bendikov@weizmann.ac.il; (U.S.) salzner@fen.bilkent.edu.tr.

$\dagger$ Weizmann Institute of Science.

$\ddagger$ Bilkent University. polymers were not fully optimized but were composed from structures of Li- and Na-doped quatermers optimized at the $\mathrm{HF}$ / STO-3G level of theory. Investigation of dications of oligothiophene up to the decamer and cyclic 12T at the AM1-based level of theory indicated that polaron pairs are more stable than bipolarons and that singlet biradical and triplet states are energetically degenerate. ${ }^{16}$ However, it is uncertain whether the theoretical methods based on the semiempirical levels used in those studies are applicable to the task of distinguishing between a bipolaron and polaron pairs. Density functional theory (DFT) calculations agree with the AM1 results in that bipolarons are intrinsically unstable with respect to separation into polarons. ${ }^{17}$ Gao et al., ${ }^{18}$ based on the DFT calculations involving dications up to $12 \mathrm{~T}^{2+}$ and employing the B3LYP hybrid functional in their singlet states, argued that polaron pairs become more stable as the oligomer length increases and gradually become the dominant state. They predicted that bipolaron and polaron pairs coexist from the hexamer to octamer and that the polaron pair becomes dominant for dications longer than octathiophene $\left(8 \mathrm{~T}^{2+}\right)$. The main difference from the AM1 results is that the transition to polaron pairs occurs at a shorter chain length. Geskin and Brédas ${ }^{19}$ compared Hartree-Fock (HF), DFT, MP2, and CAS results on the stabilities of bipolarons with those on singlet and triplet polaron pairs. It emerged that inclusion of $50 \%$ HF exchange in the DFT functional shifts the balance closer to the HF prediction of bipolarons. A triplet ground state was predicted for oligothiophenes longer than octathiophene, although they point out that with electron correlation properly taken into account the ground state might become singlet. Recently, Casanovas and Alemán studied dication oligomers of pyrrole, thiophene, phosphole, 3,4-ethylenedioxythiophene (EDOT), and 3,4-ethylenedithiafurane up to the octamer with DFT employing the B3PW91 functional. ${ }^{20}$ Polymer properties were predicted by linear extrapolation. Surprisingly, triplet ground states were predicted for oligomers longer than 8-11 rings by extrapolation from the singlet-triplet gaps of shorter oligomers. We have recently shown that, at the B3LYP level, a triplet is never the pure ground state for oligothiophene 
dications of any length with singlet and triplet states degenerated for long oligothiophene dications. ${ }^{21}$ Although for short oligomer dications (up to $10 \mathrm{~T}^{2+}$ ) the bipolaron contribution is significant, the contribution of the polaron pair state is dominant for long oligothiophene dications (up to the 50-mer, 50T ${ }^{2+}$ ). ${ }^{21}$ For all oligothiophene dications up to the 20 -mer, the singlet state was found to be the ground state. We have also shown that the singlet polaron pair state is the preferred electronic state for long oligothiophene polycations (up to $50 \mathrm{~T}^{n+}$ )..$^{22}$

As revealed by the above discussion, the balance between different states of doped polymers in the gas phase is delicate. Thus, counterions may have a major influence on the polaronbipolaron equilibrium, as was recently suggested in an experimental investigation. ${ }^{23}$ Most of the theoretical studies are limited to bare oligothiophene cations in the gas phase; however such gas phase calculations are far from the real systems. For instance, in the gas phase oligothiophene dications are very unstable toward disproportionation into two oligothiophene cation radicals even for very long oligomers (for example, the disproportionation energy of $20 \mathrm{~T}^{2+}$ is $\left.24 \mathrm{kcal} / \mathrm{mol}\right){ }^{21}$ Theoretical results on polaron-bipolaron equilibria that include dopants have been reported although the dopant was strongly bound to the oligomer chain $(\mathrm{C}-\mathrm{Cl}$ bond length is $1.99 \AA)$, thus preventing polaron pair formation. ${ }^{24}$ Investigation of the soliton widths in polyacetylene revealed that $\mathrm{Li}^{+}$and $\mathrm{Cl}^{-}$counterions lead to a decrease in defect size, whereas differences between $\mathrm{HF}, \mathrm{MP} 2$, and DFT geometries practically vanish in the presence of counterions. ${ }^{25}$ Recent work evaluated the interactions of the $\mathrm{PF}_{6}{ }^{-}$anion as a dopant on charged oligothiophenes and their dimers (up to quaterthiophene). ${ }^{26}$ Because the oligomers studied were very short, the polaron-bipolaron equilibrium and different electronic states were not investigated. ${ }^{26}$ The effect of counterions on the UV/vis spectra of oligothiophene monocations has been studied at the TDDFT level of theory. ${ }^{27}$ It was shown that the decrease in defect size due to counterions has very little effect on the first two absorption energies.

In our previous work, ${ }^{21}$ we studied long oligothiophene dications using DFT methods. However, for a thorough understanding of the nature of charge carriers we still need a more realistic model that must include the doping ions. Polythiophenes are semiconductors that become highly conductive in doped form. The doping-undoping process is central to their application in devices, although the effects of the dopant on organic electronic materials are poorly understood. Here, we report DFT studies of divalent oligothiophene salts (that is, oligothiophene dications with two dopant ions) of different lengths (up to the 20-mer) carrying two counterions $\left(\mathrm{Cl}_{3}{ }^{-}\right)$that are not covalently bound to the oligothiophene chain. We provide evidences for a change of the ground electronic state from the bipolaron for short oligothiophene salts to a polaron pair for long oligothiophene salts. Combining this study with our earlier work, ${ }^{21}$ we span the description of the electronic structure possibilities for real doped poly- and oligothiophenes by delineating the structures of oligothiophene divalent salts in the gas phase (neglecting solvent effects) at one end of the scale and of bare dications at the other end of the scale.

\section{Theoretical Methods}

Oligothiophenes with two counterions per chain (oligothiophene divalent salts) isomers are denoted as $n \mathrm{~T}^{2+}\left(\mathrm{Cl}_{3}{ }^{-}\right)_{2}$, where $n$ represents the number of oligothiophene rings $(n=8$, 12 , or 20$)$. When the focus is on the exact location of the counterions on the chain, the notation $n \mathrm{~T}^{2+}\left(\mathrm{Cl}_{3}{ }^{-}(m)\right)_{2}$, is used, where $m$ represents the oligothiophene ring number, counted

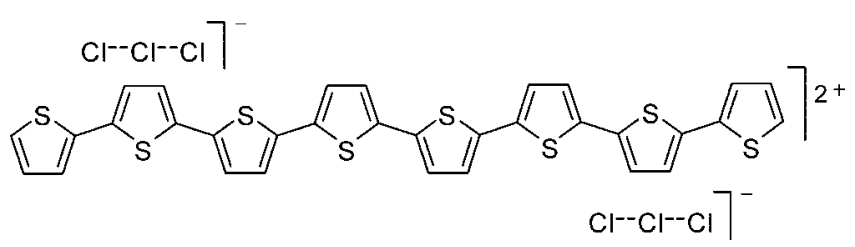

Figure 1. Example of $\mathrm{nT}^{2+}\left(\mathrm{Cl}_{3}{ }^{-}\right)_{2}(\mathrm{~m})$ oligothiophene divalent salt where $n=8$ and $m=2$.

from each end, that bears each of two symmetrically placed $\mathrm{Cl}_{3}{ }^{-}$counterions (Figure 1 ). We selected $\mathrm{Cl}_{3}{ }^{-}$counterions as the dopant because a previous theoretical study ${ }^{27}$ has shown that $\mathrm{Cl}_{3}{ }^{-}$does not form covalent bonds with oligothiophenes even in the gas phase. The charge transfer from the oligothiophene backbone to the $\mathrm{Cl}_{3}{ }^{-}$counterion at -0.89 to -0.91 el is close to one unit charge. The $\mathrm{Cl}_{3}{ }^{-}$counterion is also relatively small, which allows calculation of long oligothiophene salts within a reasonable amount of computational time.

All calculations were carried out using the Gaussian 03 series of programs. ${ }^{28}$ The geometries of the oligomers were fully optimized using hybrid DFT with Becke's three-parameter exchange functional combined with the LYP correlation functional (B3LYP), and the 6-31G(d) basis set. ${ }^{29}$ No symmetry constraints were used in geometry optimizations; however all optimized structures are practically planar. Frequency calculations were performed only for $8 \mathrm{~T}^{+} \mathrm{Cl}_{3}{ }^{-}$and $8 \mathrm{~T}^{+} \mathrm{Br}_{3}{ }^{-}$isomers (see Supporting Information). Calculations for singlet states were performed using spin-restricted and spin-unrestricted wave functions. Spin-unrestricted singlet states were calculated using the broken symmetry DFT method with the guess=(mix,always) keyword to generate an appropriate guess for the UDFT calculations. We note that the broken symmetry B3LYP method considers mixing of closed-shell and open-shell structures. ${ }^{30}$ Therefore, UB3LYP calculations always yield a solution which has a partially closed-shell structure mixed with an open-shell structure. The results obtained at the RB3LYP level correspond to a bipolaron state and the results obtained at the UB3LYP level correspond to a mixture of bipolaron and polaron pair states. Existence of the broken symmetry open-shell solution relates to the contribution from polaron pair state. In addition, triplet states were calculated for selected isomers.

Mulliken population analysis (MPA) was used to calculate the charges (summarized for thiophene rings) of the oligothiophene salts at B3LYP/6-31G(d). We have shown previously that natural population analysis (NPA) and MPA predict similar charge distributions in oligothiophene dications. ${ }^{21}$

As outlined in the introduction, the choice of theoretical level may influence results regarding polaron pair-bipolaron equilibrium. We note that with pure DFT, such as BLYP/6-31G(d), wave function instability and the onset of a contribution from polaron-pair structures are predicted at longer chain lengths than those found by hybrid DFT methods, such as B3LYP. ${ }^{21}$ However, for some long conjugated systems where the results can be compared with experimental data the B3LYP functional gives the best results. ${ }^{31}$ We believe that DFT with hybrid functionals is the best method available for long conjugated systems, as it is not possible to perform benchmark calculations with high-level ab initio methods for systems of the required size. ${ }^{32}$ Concerns regarding DFT for conjugated systems are that DFT tends to overestimate conjugation, underestimate band gaps, ${ }^{33}$ overestimate defect sizes,${ }^{34}$ and that hybrid functionals may lead to significant spin-contamination in open-shell systems. However, it has been shown that although the difference between defect sizes at different levels of theory is large, the 
TABLE 1: Relative Energies (kcal/mol) for Oligothiophene Divalent Salts $\left(8 \mathrm{~T}^{2+}\left(\mathrm{Cl}_{3}{ }^{-}(\mathrm{m})\right)_{2}, \mathrm{12T}^{2+}\left(\mathrm{Cl}_{3}{ }^{-}(\mathrm{m})\right)_{2}\right.$, and $\left.{ }_{20 T^{2+}}\left(\mathrm{Cl}_{3}{ }^{-}(m)\right)_{2}\right)$ in Spin-Restricted Singlet $(R)$, Spin-Unrestricted Singlet $(U)$, and Triplet $(T)$ States at B3LYP/6-31G(d) and $S^{2}$ Values for Spin-Unrestricted Singlet States $^{a}$

\begin{tabular}{|c|c|c|c|c|}
\hline$m$ & $R$ & $U$ & $S^{2}$ value $^{b}$ & $T^{a}$ \\
\hline \multicolumn{5}{|c|}{$8 \mathrm{~T}^{2+}\left(\mathrm{Cl}_{3}^{-}(m)\right)_{2}$} \\
\hline 2 & 7.7 & 5.8 & $0.80(0.29)$ & \\
\hline 3 & 1.4 & 1.1 & $0.43(0.06)$ & \\
\hline 4 & 0 & & & 8.4 \\
\hline \multicolumn{5}{|c|}{$12 \mathrm{~T}^{2+}\left(\mathrm{Cl}_{3}^{-}(m)\right)_{2}$} \\
\hline 2 & 13.4 & 6.0 & $1.05(0.50)$ & \\
\hline 3 & 7.3 & 1.7 & $1.02(0.49)$ & \\
\hline 4 & 3.5 & 0.5 & $0.93(0.41)$ & \\
\hline 5 & 0.7 & 0 & $0.61(0.14)$ & 3.1 \\
\hline 6 & 0.4 & & & \\
\hline \multicolumn{5}{|c|}{$20 \mathrm{~T}^{2+}\left(\mathrm{Cl}_{3}^{-}(m)\right)_{2}$} \\
\hline 2 & 16.0 & 6.4 & $1.05(0.44)$ & \\
\hline 3 & 11.3 & 2.1 & $1.05(0.45)$ & \\
\hline 4 & 9.4 & 0.7 & $1.06(0.46)$ & \\
\hline 5 & 8.0 & 0.1 & $1.06(0.47)$ & 0.03 \\
\hline 6 & 6.9 & 0 & $0.95(0.40)$ & 0.09 \\
\hline 7 & 5.5 & 0.2 & $1.03(0.48)$ & \\
\hline 8 & 3.5 & 0.5 & $0.95(0.40)$ & \\
\hline 9 & 1.7 & 0.9 & $0.64(0.15)$ & \\
\hline 10 & 1.6 & 1.6 & $0.03(0)$ & 5.0 \\
\hline
\end{tabular}

${ }^{a}$ The energies of all isomers were calculated relative to the most stable isomer, while the energies of triplet states were calculated relative to the corresponding singlet states. ${ }^{b} S^{2}$ values after annihilation of the first spin component are given in parentheses.

reorganization energies required to change the defect size are very small. ${ }^{35}$ In addition, the B3LYP/6-31G(d) level correctly predicts the band gap of conjugated polymers, in particular that of polythiophene, ${ }^{36,37}$ and DFT defect sizes match those at the MP2 level in the presence of counterions. ${ }^{25}$ Furthermore, spincontamination is virtually absent for open-shell cation radicals of oligothiophenes ${ }^{27}$ and spin-contamination is small for openshell dications of oligothiophenes. ${ }^{21}$ So, we believe that DFT is the best practical theoretical level at which to study the systems considered in this paper. ${ }^{38}$ Polaron-pair states can be viewed as biradicals. Recent discussion in the literature regarding the applicability of DFT as a tool for studying biradicals concluded that UDFT is the best method for studying large systems where high-level ab initio calculations are impractical. ${ }^{39}$ For a more detailed discussion see ref 40 .

\section{Results}

Relative Energies of Divalent Salt Isomers. Structures of oligothiophene divalent salts $\left(8 \mathrm{~T}^{2+}\left(\mathrm{Cl}_{3}{ }^{-}(m)\right)_{2}, \quad m=2-4\right.$; $12 \mathrm{~T}^{2+}\left(\mathrm{Cl}_{3}{ }^{-}(m)\right)_{2}, m=2-6$; and $\left.20 \mathrm{~T}^{2+}\left(\mathrm{Cl}_{3}{ }^{-}(m)\right)_{2}, m=2-10\right)$ were optimized in conformations where counterions are in-plane with the oligothiophene chains. ${ }^{41}$ The energies of all oligothiophene divalent salt isomers were calculated relative to the most stable isomer, while the energies of triplet states were calculated relative to the corresponding singlet states. The results are summarized in Table 1 and the most stable isomers are shown in Figure 2. Similarly to the monovalents salts, $\mathrm{Cl}_{3}{ }^{-}$counterions in divalent salts are not covalently bound to the oligothiophene backbone (Figure 2). Shortest $\mathrm{H} \cdots \mathrm{Cl}$ distances of 2.55-2.61 remain practically unchanged with increasing oligothiophene chain length in all divalent salts. ${ }^{42}$

For short oligothiophene divalent salts, such as $8 \mathrm{~T}^{2+}\left(\mathrm{Cl}_{3}{ }^{-}\right)_{2}$, the most stable isomer is $8 \mathrm{~T}^{2+}\left(\mathrm{Cl}_{3}{ }^{-}(4)\right)_{2}$ with the counterions
SCHEME 1: Structure of the Aromatic (Left) and the Quinoid (Right) Form of Oligothiophene
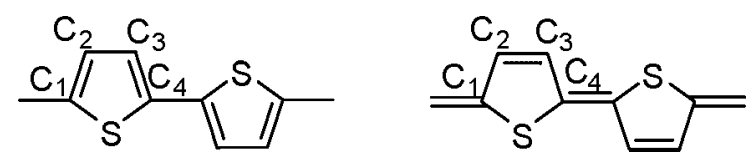

located in the middle of the chain (Table 1 and Figure 2a). For the medium-sized salts, such as $12 \mathrm{~T}^{2+}\left(\mathrm{Cl}_{3}{ }^{-}\right)_{2}$, the most stable isomer is $12 \mathrm{~T}^{2+}\left(\mathrm{Cl}_{3}{ }^{-}(5)\right)_{2}$ (Figure 2b) where the counterions are located one ring from the center. The restricted wave function is unstable for this structure and optimization at UB3LYP/6-31G(d) leads to a $0.7 \mathrm{kcal} / \mathrm{mol}$ lower energy (Table 1). ${ }^{43}$ Wave function instability that leads to broken symmetry open-shell solution points out to the contribution of the polaron pair state to the electronic structure of medium-sized salts. The lowest energy structure at RB3LYP is that of $12 \mathrm{~T}^{2+}\left(\mathrm{Cl}_{3}{ }^{-}(6)\right)_{2}$, which is only $0.4 \mathrm{kcal} / \mathrm{mol}$ above the minimum. There are a number of isomers with energies very close to that of $12 \mathrm{~T}^{2+}\left(\mathrm{Cl}_{3}{ }^{-}(5)\right)_{2}$, namely $12 \mathrm{~T}^{2+}\left(\mathrm{Cl}_{3}{ }^{-}(6)\right)_{2}(+0.4 \mathrm{kcal} / \mathrm{mol})$ and $12 \mathrm{~T}^{2+}\left(\mathrm{Cl}_{3}{ }^{-}(4)\right)_{2}(+0.5 \mathrm{kcal} / \mathrm{mol})$, indicating that the potential energy surface for the movement of counterions along the oligothiophene chain is flat. The most stable isomer of $20 \mathrm{~T}^{2+}\left(\mathrm{Cl}_{3}{ }^{-}\right)_{2}$ salts is $20 \mathrm{~T}^{2+}\left(\mathrm{Cl}_{3}{ }^{-}(6)\right)_{2}$ (Figure 2c) with 8 thiophene rings between the two counterions and 5 thiophene units to the termini. The energy difference between the spinrestricted state and the more stable spin-unrestricted state of $20 \mathrm{~T}^{2+}\left(\mathrm{Cl}_{3}{ }^{-}(6)\right)_{2}$ is significant $(6.9 \mathrm{kcal} / \mathrm{mol})$. If only spinrestricted wave functions are considered (corresponding to bipolaron states) then the lowest energy structure of $20 \mathrm{~T}^{2+}\left(\mathrm{Cl}_{3}{ }^{-}\right)_{2}$ is $20 \mathrm{~T}^{2+}\left(\mathrm{Cl}_{3}{ }^{-}(10)\right)_{2}$, which is $1.6 \mathrm{kcal} / \mathrm{mol}$ higher in energy than the minimum energy spin-unrestricted structure. The difference between the lowest energies at RB3LYP/ 6-31G(d) and UB3LYP/6-31G(d) is significantly larger for $20 \mathrm{~T}^{2+}\left(\mathrm{Cl}_{3}{ }^{-}\right)_{2}$ than for $12 \mathrm{~T}^{2+}\left(\mathrm{Cl}_{3}{ }^{-}\right)_{2}$. We note that the expectation values of the spin operator $\left(S^{2}\right.$ values) before annihilation (Table 1) are less than 1 for $8 \mathrm{~T}^{2+}\left(\mathrm{Cl}_{3}{ }^{-}\right)_{2}$ isomers and for some of the isomers of $12 \mathrm{~T}^{2+}\left(\mathrm{Cl}_{3}{ }^{-}\right)_{2}$ and $20 \mathrm{~T}^{2+}\left(\mathrm{Cl}_{3}{ }^{-}\right)_{2}$. The $S^{2}$ value stays around 1 (at $0.93-1.06$ ) for most of the isomers of $12 \mathrm{~T}^{2+}\left(\mathrm{Cl}_{3}{ }^{-}\right)_{2}$ and $20 \mathrm{~T}^{2+}\left(\mathrm{Cl}_{3}{ }^{-}\right)_{2}$, which indicates that the electronic states should be quite close to 50:50 mixtures of the singlet $\left(S^{2}=0\right)$ and triplet $\left(S^{2}=2\right)$ states.

Geometries of Oligothiophene Divalent Salts. The geometries of the oligothiophene backbones as a function of the position of the counterions for oligothiophene divalent salts and for the corresponding oligothiophene dications ${ }^{21}$ are presented in Figure 3 as a bond length alternation (BLA) pattern (the complete XYZ coordinates of all calculated salts are given in Supporting Information). Charged $\pi$-conjugated systems such as oligothiophenes tend to have partially quinoid geometries in contrast to neutral $\pi$-conjugated systems, which have aromatic structures (Scheme 1). ${ }^{22,36,44}$

The BLA pattern for $8 \mathrm{~T}^{2+}\left(\mathrm{Cl}_{3}{ }^{-}(4)\right)_{2}$ has a strongly quinoid character for the central four rings and an aromatic character for the terminal rings (Figure 3a). The interring $\mathrm{C}-\mathrm{C}$ bond between the central rings is especially short, being $1.388 \AA$. For all other $8 \mathrm{~T}^{2+}\left(\mathrm{Cl}_{3}{ }^{-}\right)_{2}$ isomers (Figure 3a), all rings are quinoid, except for the terminal rings. Interestingly, $8 \mathrm{~T}^{2+}\left(\mathrm{Cl}_{3}-(2)\right)_{2}$ has the most similar BLA pattern to that of the bare dication.

For the minimal energy isomer of $12 \mathrm{~T}^{2+}\left(\mathrm{Cl}_{3}{ }^{-}\right)_{2}$, namely $12 \mathrm{~T}^{2+}\left(\mathrm{Cl}_{3}{ }^{-}(5)\right)_{2}$, the six central rings are quinoid and three terminal rings from each side are aromatic. For $12 \mathrm{~T}^{2+}\left(\mathrm{Cl}_{3}{ }^{-}(2)\right)_{2}$ (Figure $3 b$ ), the rings close to the counterions are quinoid, and 

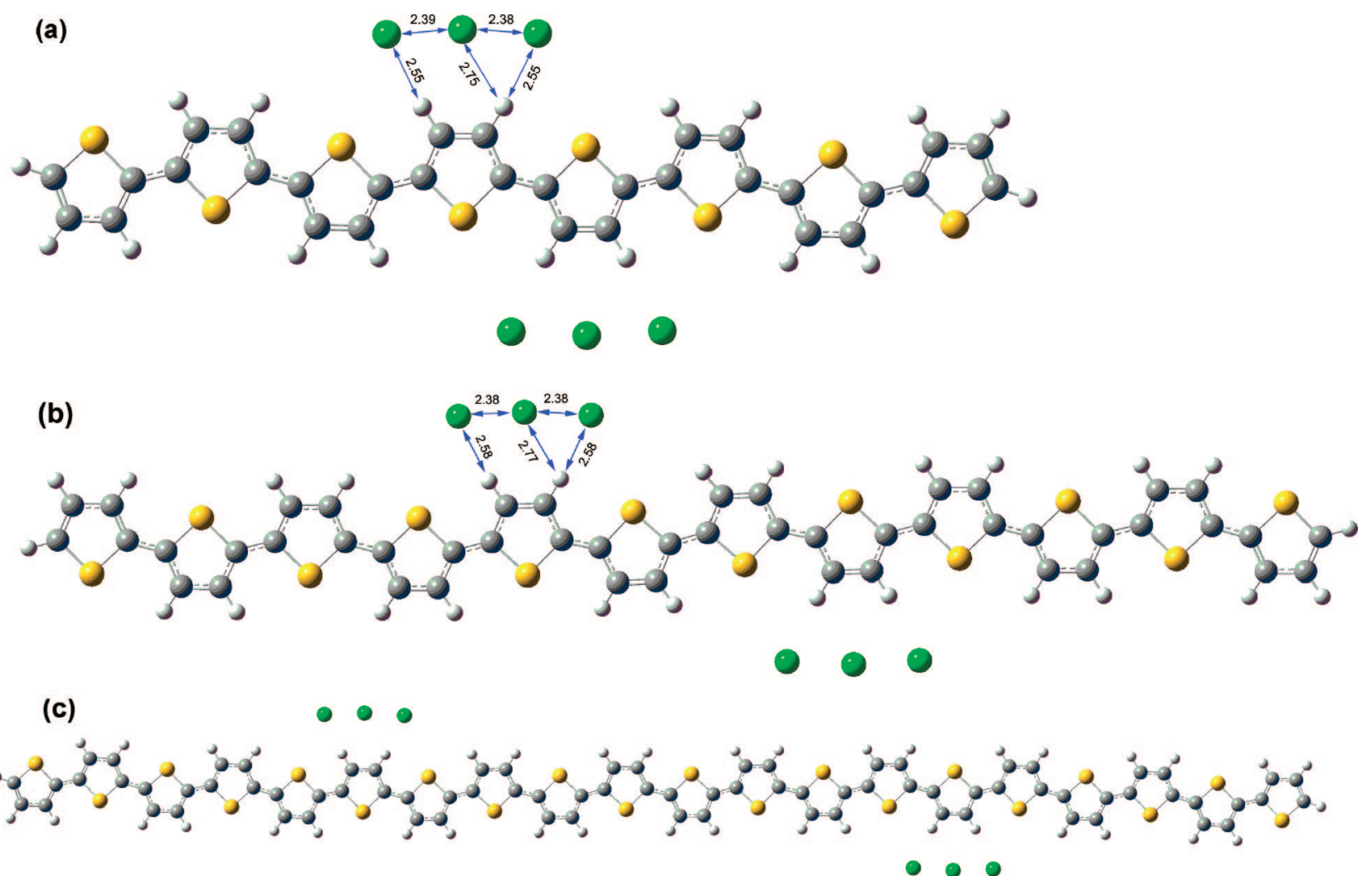

Figure 2. Most stable isomers of (a) $8 \mathrm{~T}^{2+}\left(\mathrm{Cl}_{3}{ }^{-}\right)_{2}$ (i.e., $\left.8 \mathrm{~T}^{2+}\left(\mathrm{Cl}_{3}{ }^{-}(4)\right)_{2}\right)$, (b) $12 \mathrm{~T}^{2+}\left(\mathrm{Cl}_{3}{ }^{-}\right)_{2}$ (i.e., $\left.12 \mathrm{~T}^{2+}\left(\mathrm{Cl}_{3}{ }^{-}(5)\right)_{2}\right)$ and (c) $20 \mathrm{~T}^{2+}\left(\mathrm{Cl}_{3}{ }^{-}\right)_{2}(\mathrm{i}$. e., $\left.20 \mathrm{~T}^{2+}\left(\mathrm{Cl}_{3}^{-}(6)\right)_{2}\right)$. All distances are given in $\AA$.
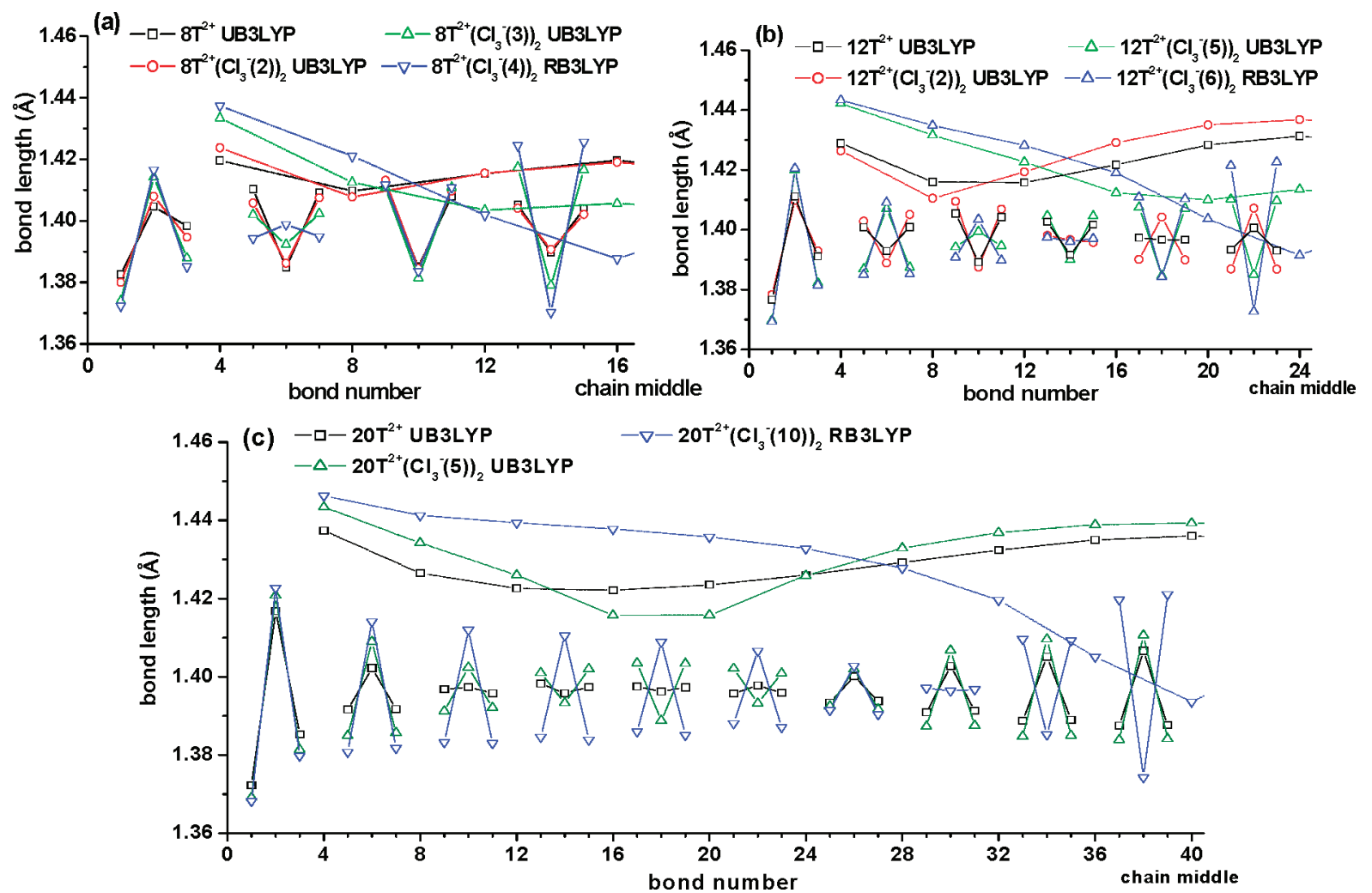

Figure 3. Bond length alternation (BLA) patterns for representative isomers of oligothiophene divalent salts and dications: (a) $8 \mathrm{~T}^{2+}\left(\mathrm{Cl}_{3}{ }^{-}\right)_{2}$, $(\mathrm{b})$ $12 \mathrm{~T}^{2+}\left(\mathrm{Cl}_{3}{ }^{-}\right)_{2}$, and (c) $20 \mathrm{~T}^{2+}\left(\mathrm{Cl}_{3}{ }^{-}\right)_{2}$. The $x$-axis is the $\mathrm{C}-\mathrm{C}$ bond number starting from one end of the conjugated chain through to the middle of that chain. A mirror image of the pattern shown in the figure is observed for the remaining half of each oligothiophene chain. The points are connected solely as a visual aid.

the central rings, which are relatively far from the counterions, are aromatic. However, the $12 \mathrm{~T}^{2+}\left(\mathrm{Cl}_{3}{ }^{-}(2)\right)_{2}$ isomer is not the minimal energy isomer for $12 \mathrm{~T}^{2+}\left(\mathrm{Cl}_{3}{ }^{-}\right)_{2}$ (see Table 1).
The BLA pattern of $20 \mathrm{~T}^{2+}\left(\mathrm{Cl}_{3}{ }^{-}(5)\right)_{2}$ shows three aromatic rings at each end of the chain, followed by three quinoid rings in each of the segments adjacent to a dopant anion (rings 4-6 
and 15-17) with eight aromatic rings located at the center of the chain (Figure 3c). ${ }^{45}$ The BLA pattern of $20 \mathrm{~T}^{2+}\left(\mathrm{Cl}_{3}{ }^{-}(10)\right)_{2}$ is characteristic of a bipolaron structure (six quinoid rings in the middle of the chain and seven aromatic rings from each side of the chain). This is as expected given the close proximity of the dopants to one another, which confines the two positive charges in the middle of the chain (see Figure 6 below). We note that this isomer is not the global minimum; rather it lies $1.6 \mathrm{kcal} / \mathrm{mol}$ above $20 \mathrm{~T}^{2+}\left(\mathrm{Cl}_{3}{ }^{-}(6)\right)_{2}$.

Frontier Molecular Orbitals of 20T, 20T ${ }^{2+}$, and $\mathbf{2 0 T}^{\mathbf{2}+}\left(\mathbf{C l}_{\mathbf{3}}{ }^{-}\right)_{2} \cdot{ }^{46}$ As expected, frontier molecular orbitals (highest occupied molecular orbital (HOMO) and lowest unoccupied molecular orbital (LUMO)) of oligothiophene dications are similar to the HOMO and HOMO-1 of the corresponding neutral oligothiophene, as exemplified in Figure 4a,b. Both the HOMO and LUMO for $20 \mathrm{~T}^{2+}$ and $20 \mathrm{~T}^{2+}\left(\mathrm{Cl}_{3}{ }^{-}\right)_{2}$ are located on the oligothiophene backbone. In $20 \mathrm{~T}^{2+}\left(\mathrm{Cl}_{3}{ }^{-}(10)\right)_{2}$ (at RB3LYP/ 6-31G(d)//RB3LYP/6-31G(d)), the HOMO has coefficients at the terminal fragments of the oligothiophene backbone with practically zero coefficients on the central rings close to the $\mathrm{Cl}_{3}{ }^{-}$counterions where most of the positive charge is located (see Figure $6 \mathrm{c}$ below). This orbital corresponds to the HOMO-1 of neutral 20T, which also has practically zero coefficients around the center of the chain (Figure 4a,c). Orbitals located on the $\mathrm{Cl}_{3}{ }^{-}$dopant are HOMO-2 and some other lower lying orbitals. The LUMO of $20 \mathrm{~T}^{2+}\left(\mathrm{Cl}_{3}{ }^{-}(10)\right)_{2}$ is similar to the HOMO of neutral 20T and is located at the center of the chain. Both the HOMO and LUMO of $20 \mathrm{~T}^{2+}\left(\mathrm{Cl}_{3}{ }^{-}(10)\right)_{2}$ are more localized compared to the HOMO-1 and HOMO, respectively, of neutral 20T. In $20 \mathrm{~T}^{2+}\left(\mathrm{Cl}_{3}{ }^{-}(5)\right)_{2}$ (at UB3LYP/6-31G(d)// UB3LYP/6-31G(d)), the $\alpha$ and $\beta$ orbitals are mirror images of each other having identical orbital energies. The HOMO of $20 \mathrm{~T}^{2+}\left(\mathrm{Cl}_{3}-(5)\right)_{2}$ is delocalized over the center and one side of the chain. Similar to that of $20 \mathrm{~T}^{2+}\left(\mathrm{Cl}_{3}{ }^{-}(10)\right)_{2}$, the LUMO of $20 \mathrm{~T}^{2+}\left(\mathrm{Cl}_{3}{ }^{-}(5)\right)_{2}$ is located on the rings close to the dopant. As expected, the shapes of the LUMOs in $20 \mathrm{~T}^{2+}\left(\mathrm{Cl}_{3}{ }^{-}(10)\right)_{2}$ and $20 \mathrm{~T}^{2+}\left(\mathrm{Cl}_{3}{ }^{-}(5)\right)_{2}$ closely resemble the charge distribution in these salts (see Figure $6 \mathrm{c}$ below) because the LUMO is the orbital from which two electrons were removed.

Singlet versus Triplet States of Divalent Salts. We have calculated triplet states (complete geometry optimizations were performed) for the most stable isomers of oligothiophene divalent salts (Table 1). For short divalent salts $\left(8 \mathrm{~T}^{2+}\left(\mathrm{Cl}_{3}{ }^{-}(4)\right)_{2}\right)$, the singlet state is $8.4 \mathrm{kcal} / \mathrm{mol}$ lower in energy than the triplet state, for $12 \mathrm{~T}^{2+}\left(\mathrm{Cl}_{3}{ }^{-}(5)\right)_{2}$ it is lower by $3.1 \mathrm{kcal} / \mathrm{mol}$, and for $20 \mathrm{~T}^{2+}\left(\mathrm{Cl}_{3}{ }^{-}(6)\right)_{2}$ the two states are practically degenerate with an energy difference of only $0.09 \mathrm{kcal} / \mathrm{mol}$. Similar to our findings regarding the bare oligothiophene dications, the energy difference between the singlet and triplet states $\left(\Delta E_{\mathrm{T}-\mathrm{S}}\right)$ decreases with increasing oligomer length, while singlet-triplet difference is smaller in bare oligothiophene dications than in divalent salts (see Supporting Information).

Relative Stability of Divalent Salts. We have estimated the stability of oligothiophene divalent salts using eqs $1-4$. The most stable isomers were used. The results obtained for salts were compared with the results obtained for bare oligothiophene dications (eqs $5^{21}$ and 6), summarized in Table 2 and plotted versus the reciprocal of oligomer chain length in Figure 5.

$$
\begin{gathered}
n \mathrm{~T}^{2+}\left(\mathrm{Cl}_{3}{ }^{-}\right)_{2}+n \mathrm{~T} \rightarrow 2 n \mathrm{~T}^{+} \mathrm{Cl}_{3}{ }^{-} \\
n \mathrm{~T}^{2+}\left(\mathrm{Cl}_{3}{ }^{-}\right)_{2}+2\left(\frac{n}{2} \mathrm{~T}\right) \rightarrow 2\left(\frac{n}{2} \mathrm{~T}^{+} \mathrm{Cl}_{3}{ }^{-}\right)+n \mathrm{~T} \\
n \mathrm{~T}^{2+}\left(\mathrm{Cl}_{3}{ }^{-}\right)_{2} \rightarrow n \mathrm{~T}^{2+}+2 \mathrm{Cl}_{3}^{-}
\end{gathered}
$$

$$
\begin{gathered}
n \mathrm{~T}^{2+}\left(\mathrm{Cl}_{3}{ }^{-}\right)_{2} \rightarrow n \mathrm{~T}+2 \mathrm{Cl}_{3} \\
n \mathrm{~T}^{2+}+n \mathrm{~T} \rightarrow 2 n \mathrm{~T}^{+\bullet} \\
n \mathrm{~T}^{2+}+2\left(\frac{n}{2} \mathrm{~T}\right) \rightarrow 2\left(\frac{n}{2} \mathrm{~T}^{+\bullet}\right)+n \mathrm{~T}
\end{gathered}
$$

In oligothiophene divalent salts, charge separation, that is, synproportionation of the dication and neutral oligomer into two cations (eq 1 and Figure 5a), is energetically preferred by only $4.5 \mathrm{kcal} / \mathrm{mol}$ for short chains $\left(8 \mathrm{~T}^{2+}\left(\mathrm{Cl}_{3}{ }^{-}\right)_{2}\right)$ and by only $0.9 \mathrm{kcal} /$ mol for long chains $\left(20 \mathrm{~T}^{2+}\left(\mathrm{Cl}_{3}{ }^{-}\right)_{2}\right)$ (Table 2). This is somewhat surprising, considering that the positive charge in oligothiophene monovalent salts (right part of eq 1) can be delocalized over the whole oligothiophene chain, while in long divalent oligothiophene salts, each of the charges resides on half of an oligothiophene chain (see charge distribution, Figure 6 below). According to eq 2 and Figure $5 \mathrm{~b}$, the short oligothiophene divalent salt $\left(8 \mathrm{~T}^{2+}\left(\mathrm{Cl}_{3}{ }^{-}\right)_{2}\right)$ is more stable (i.e., separation of two charges is thermodynamically unfavored, by $7.0 \mathrm{kcal} / \mathrm{mol}$, Table 2) than two oligothiophene monovalent salts of half the chain length. However, for long oligothiophene divalent salts $\left(20 \mathrm{~T}^{2+}\left(\mathrm{Cl}_{3}{ }^{-}\right)_{2}\right)$, eq 2 is thermoneutral. In the case of bare oligothiophene dications, separation of a dication into two cation radicals of half the length (eq 6) is always energetically preferred (by $10.4-25.5 \mathrm{kcal} / \mathrm{mol}$, Table 2) for the oligothiophenes studied, however extrapolation to infinite oligomer length results in thermoneutral reactions (Figure 5b). Dissociation of oligothiophene divalent salts into charged species, namely a bare oligothiophene dication and two $\mathrm{Cl}_{3}{ }^{-}$anions (eq 3), requires very high energy in the gas phase $(112.6-156.3 \mathrm{kcal} / \mathrm{mol}$ for the oligomers studied, Table 2). The extrapolation of eq 3 to infinite oligothiophene length (Figure $5 \mathrm{c}$ ) results in a value of $83.7 \mathrm{kcal} / \mathrm{mol}$; this energy probably corresponds to twice the intrinsic attraction energy between the positive charge on the oligothiophene chain and the $\mathrm{Cl}_{3}{ }^{-}$anion. Dissociation of divalent oligothiophene salts into neutral species, namely oligothiophene and two $\mathrm{Cl}_{3}{ }^{\circ}$ radicals (eq 4), requires significantly less energy in the gas phase $(63.7-69.1 \mathrm{kcal} / \mathrm{mol}$ for the oligomers studied, Table 2) than that required by eq 3 . This dissociation energy increases with increasing chain length (Table 2). Extrapolation of eq 4 to infinite oligothiophene length (Figure $5 \mathrm{c}$ ) results in a value of $72.6 \mathrm{kcal} / \mathrm{mol}$.

Charge Distribution in Oligothiophene Divalent Salts. Using $\mathrm{Cl}_{3}{ }^{-}$as a dopant results in practically complete transfer of an electron (precisely 0.9 el) from the oligothiophene backbone to each of the two dopants in oligothiophene divalent salts. Mulliken charge distribution patterns (Figure 6, charges are summarized for each thiophene ring) demonstrate that the positive charges are partly localized on the rings close to the counterions (which are the regions where the BLA pattern usually shows a quinoid structure). When negatively charged counterions are located near the middle rings of the oligothiophene chain, the positive charges are also localized around those rings, with central rings carrying similar amounts of charge (up to $0.33-0.36$ units) regardless of $n$ ( $n=8,12$ and 20, Figure $6)$. When the counterions are gradually shifted toward the chain edges, more charge delocalization is observed with maximal charges always located on the rings adjacent to the negative counterions, which leads to some charge localization around the dopant region. Interestingly, the positive charge located on the rings adjacent to the dopant is practically the same in all isomers (except for isomers where the counterions are located at the center), being $\sim 0.27 \mathrm{el}$. for $8 \mathrm{~T}^{2+}\left(\mathrm{Cl}_{3}{ }^{-}\right)_{2}$ isomers, $\sim 0.25$ el. for $12 \mathrm{~T}^{2+}\left(\mathrm{Cl}_{3}{ }^{-}\right)_{2}$, and $\sim 0.22$ el. for $20 \mathrm{~T}^{2+}\left(\mathrm{Cl}_{3}{ }^{-}\right)_{2}$ isomers (Figure 6). For $8 \mathrm{~T}^{2+}\left(\mathrm{Cl}_{3}{ }^{-}\right)_{2}$ and $12 \mathrm{~T}^{2+}\left(\mathrm{Cl}_{3}{ }^{-}\right)_{2}$ isomers in which 
a) $20 \mathrm{~T}$

LUMO

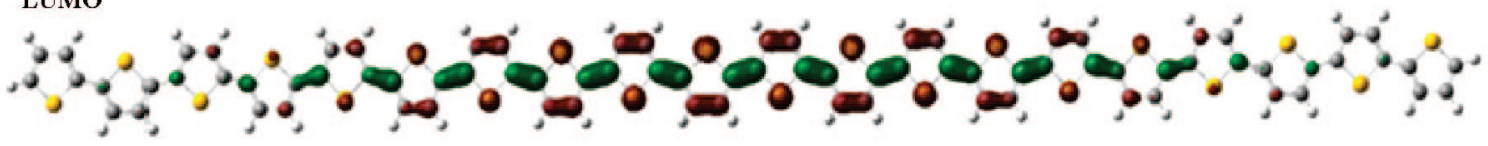

HOMO

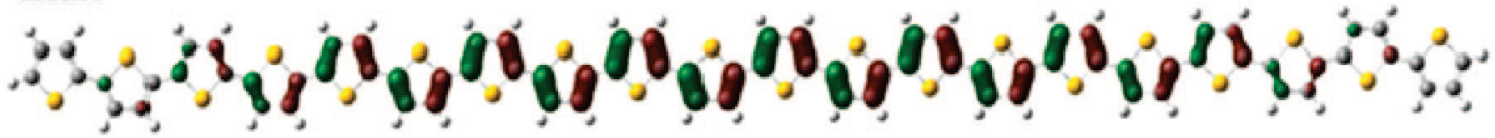

HOMO-1

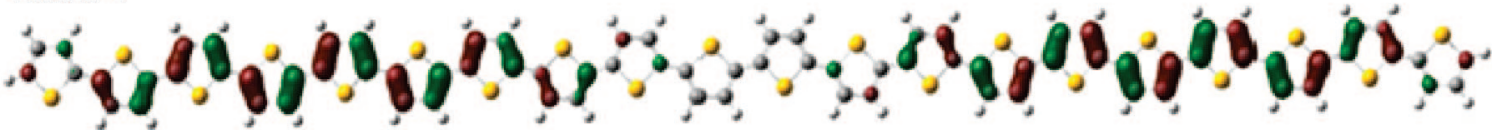

b) $20 \mathrm{~T}^{2+}$

LUMO

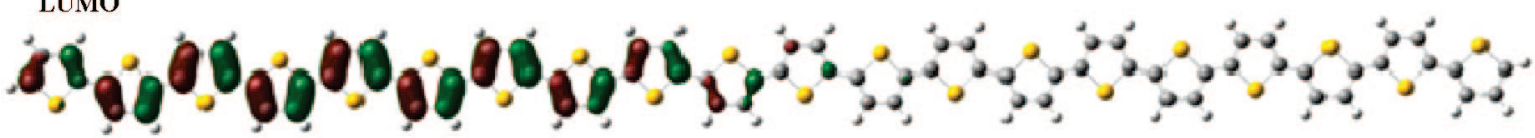

Hомо

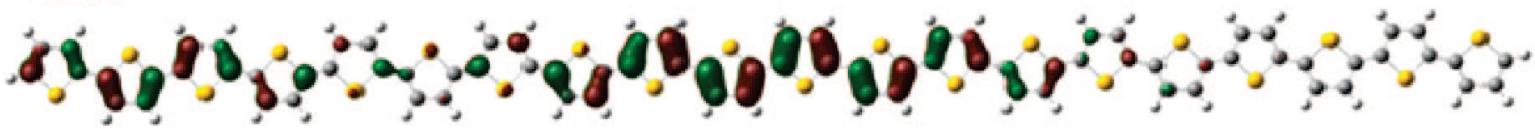

c) $20 \mathrm{~T}^{2+}\left(\mathrm{Cl}_{3}^{-}(10)\right)_{2}$

LUMO

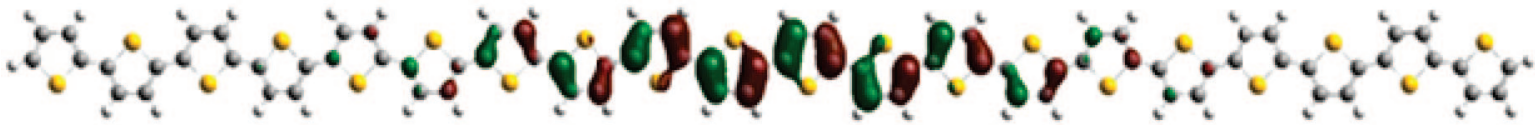

$\bullet \bullet$

HOMO

"

-

d) $20 \mathrm{~T}^{2+}\left(\mathrm{Cl}_{3}^{-}(5)\right)_{2}$
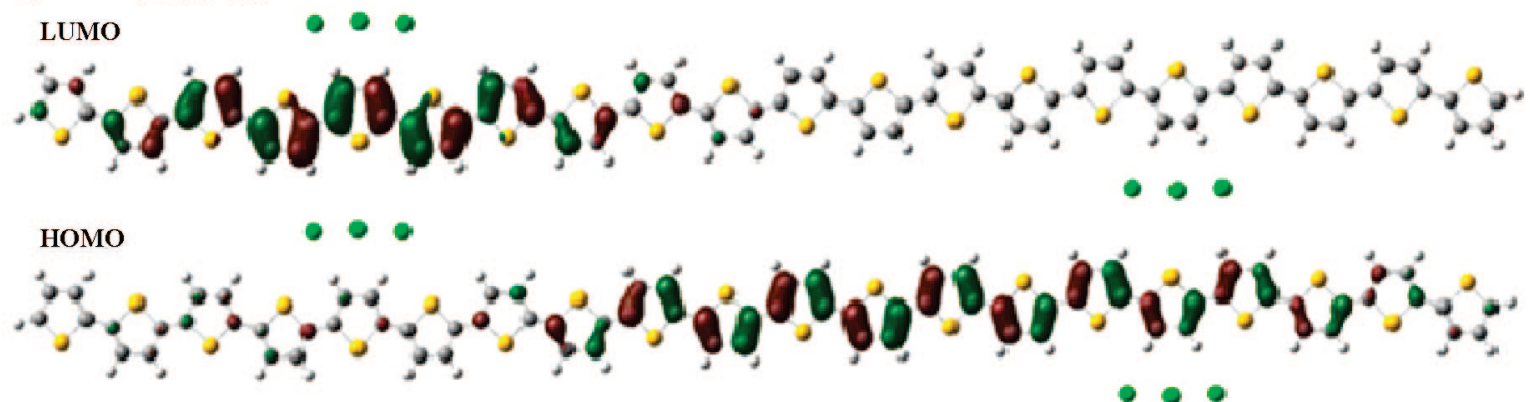

Figure 4. Frontier molecular orbitals of (a) $20 \mathrm{~T}$ at RB3LYP/6-31G(d), (b) $20 \mathrm{~T}^{2+}$ at $\mathrm{UB} 3 \mathrm{LYP} / 6-31 \mathrm{G}(\mathrm{d})$, (c) $20 \mathrm{~T}^{2+}\left(\mathrm{Cl}_{3}{ }^{-}(10)\right)_{2}$ at $\mathrm{RB} 3 \mathrm{LYP} /$ 6-31G(d), and (d) $20 \mathrm{~T}^{2+}\left(\mathrm{Cl}_{3}{ }^{-}(5)\right)_{2}$ at UB3LYP/6-31G(d) (for calculations at the UB3LYP/6-31G(d) level, only the $\alpha$ orbitals are shown; $\beta$ orbitals are mirror images of the $\alpha$ orbitals, having the same shape and energy). ${ }^{47}$

the counterions are positioned close to the chain edges (Figure $6 a, b$, respectively), the charge separation is well observed as a dip in the plot, whereas middle rings still carry some amount of positive charge. For $20 \mathrm{~T}^{2+}\left(\mathrm{Cl}_{3}{ }^{-}\right)_{2}$, charge separation is obtained even for the $20 \mathrm{~T}^{2+}\left(\mathrm{Cl}_{3}{ }^{-}(7)\right)_{2}$ isomer (see Supporting Information) and all patterns (except for isomer $\left.20 \mathrm{~T}^{2+}\left(\mathrm{Cl}_{3}{ }^{-}(10)\right)_{2}\right)$ represent two separated polarons (Figure 6c). The charge separation is practically complete (charges on the middle rings are almost zero) for the $20 \mathrm{~T}^{2+}\left(\mathrm{Cl}_{3}{ }^{-}(3)\right)_{2}$ isomer (see Supporting Information). In $20 \mathrm{~T}^{2+}\left(\mathrm{Cl}_{3}{ }^{-}(5)\right)_{2}$ (Figure 6c), charge separation is clearly observed with charges on the central rings as small as $0.03 \mathrm{el}$. 
(a)

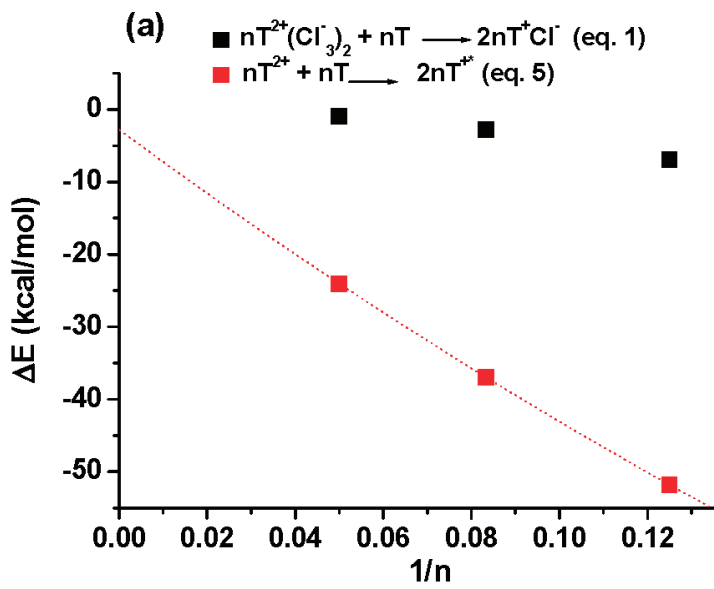

(b) $\quad n \mathrm{~T}^{2+}\left(\mathrm{Cl}_{3}\right)_{2}+2(\mathrm{n} / 2 \mathrm{~T}) \rightarrow 2\left(\mathrm{n} / 2 \mathrm{~T}^{\mathrm{C}} \mathrm{Cl} \mathrm{C}_{3}\right)+\mathrm{nT}$ (eq. 2)

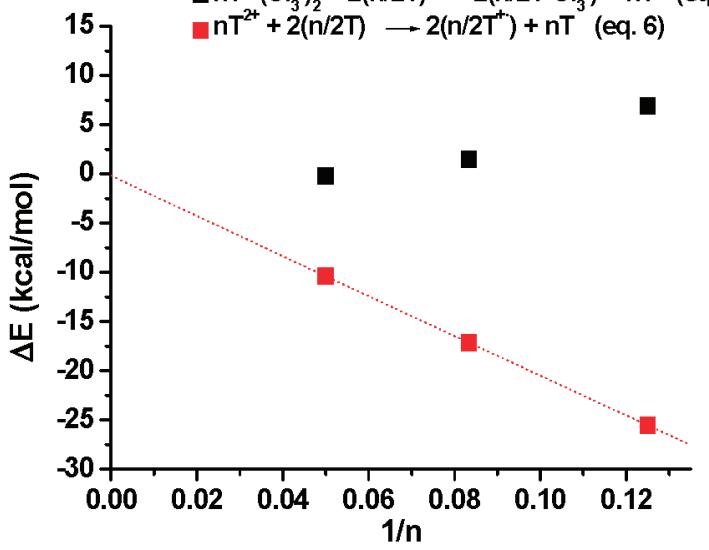

(c)

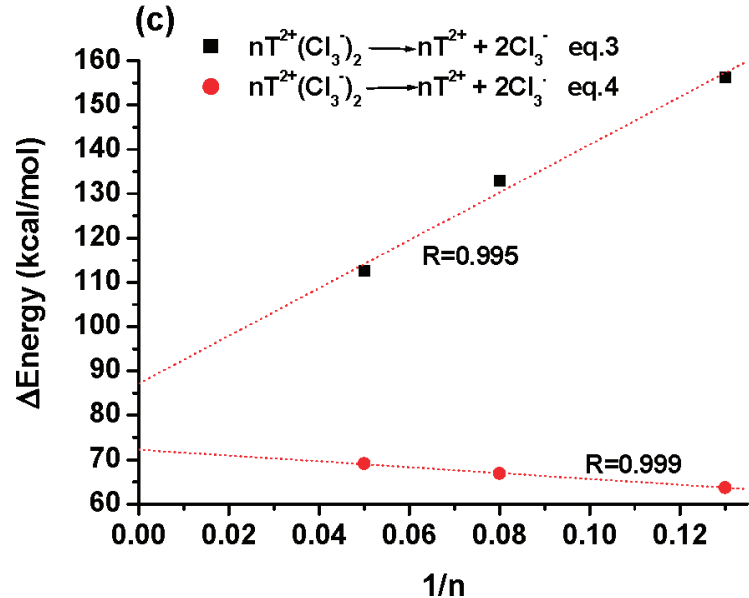

Figure 5. Energies of (a) eqs 1 and 5, (b) eqs 2 and 6, and (c) eqs 3 and 4 versus the inverse of oligothiophene chain length. Linear regression was used to connect the points from eqs 3-6.

TABLE 2: Calculated Energies (in $\mathrm{kcal} / \mathrm{mol}$ at B3LYP/ 6-31G(d)) for Equations 1-6

\begin{tabular}{lrrrrrr}
\hline$n$ & eq 1 & eq 2 & eq 3 & eq 4 & eq 5 & eq 6 \\
\hline 8 & -4.5 & 7.0 & 156.3 & 63.7 & -53.4 & -25.5 \\
12 & -2.8 & 1.5 & 132.9 & 66.9 & -37.0 & -17.1 \\
20 & -0.9 & -0.2 & 112.6 & 69.1 & -24.0 & -10.4
\end{tabular}

\section{Discussion}

Conducting polymers usually do not have high molecular weights. ${ }^{48}$ The longest oligomer studied in this paper, $20 \mathrm{~T}^{2+}\left(\mathrm{Cl}_{3}{ }^{-}\right)_{2}$, has $M_{\mathrm{w}}=1855 \mathrm{~g} / \mathrm{mol}$ and sufficient conjugation length (the optical absorption of 20T is expected to be not much
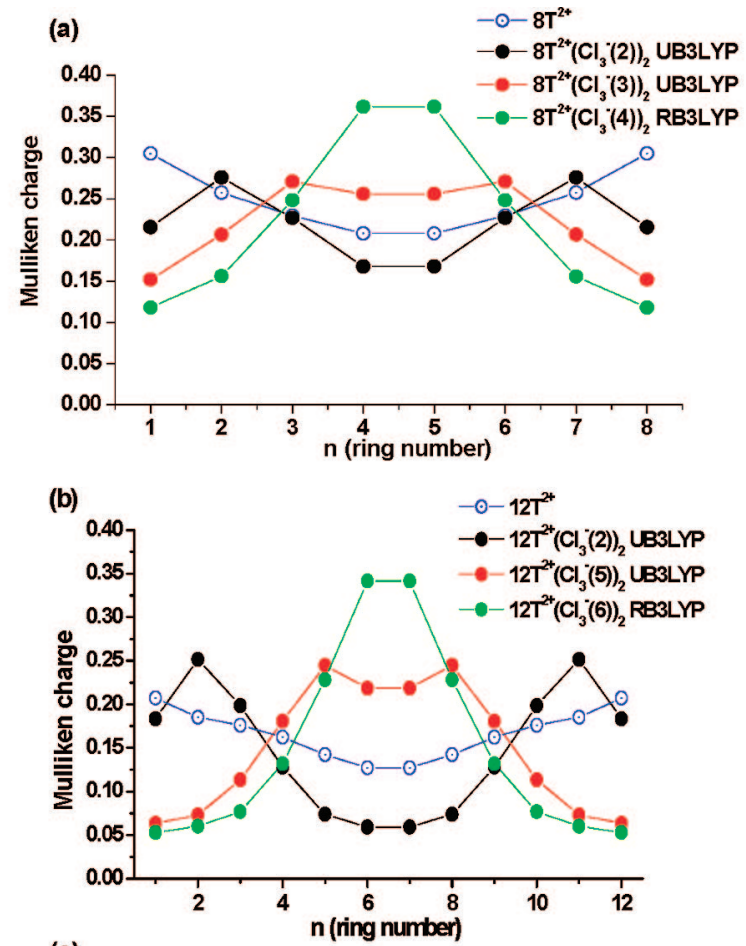

(c)

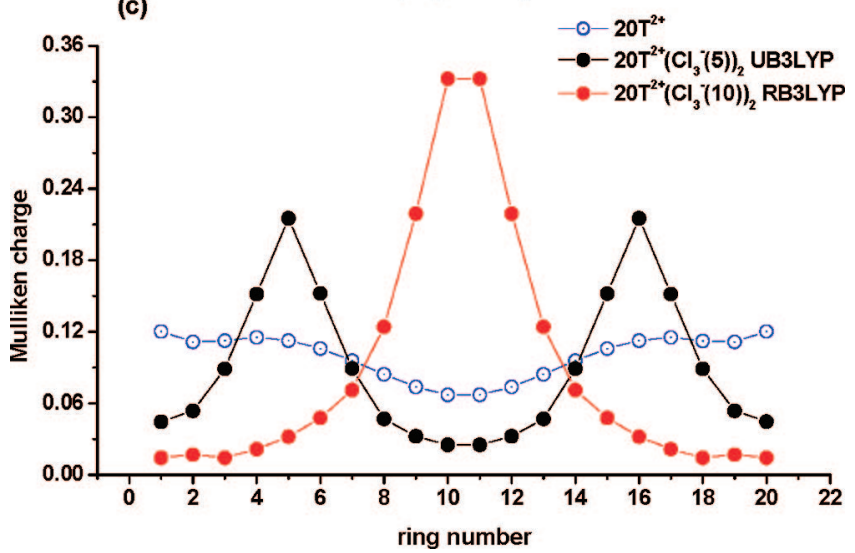

Figure 6. Mulliken charge distribution summarized for thiophene rings (at B3LYP/6-31G(d)) for (a) $8 \mathrm{~T}^{2+}\left(\mathrm{Cl}_{3}{ }^{-}\right)_{2}$, (b) $12 \mathrm{~T}^{2+}\left(\mathrm{Cl}_{3}{ }^{-}\right)_{2}$, and (c) $20 \mathrm{~T}^{2+}\left(\mathrm{Cl}_{3}{ }^{-}\right)_{2}$ isomers.

different from that of polythiophene $\left.{ }^{37}\right)$. Therefore, $20 \mathrm{~T}^{2+}\left(\mathrm{Cl}_{3}{ }^{-}\right)_{2}$ can be considered a reasonable model for short chains of polythiophene ${ }^{49}$ For oligothiophene divalent salts, our calculations correspond to doping levels ranging from $10 \%$ (in the case of $\left.20 \mathrm{~T}^{2+}\left(\mathrm{Cl}_{3}{ }^{-}\right)_{2}\right)$ to $25 \%$ (in the case of $\left.8 \mathrm{~T}^{2+}\left(\mathrm{Cl}_{3}{ }^{-}\right)_{2}\right)$. In the case of a high doping level, our results are applicable to experimentally studied highly doped polythiophenes and oligothiophenes, whereas a lower doping level corresponds to medium-doped polythiophenes.

In the oligothiophene salts, restricted wave functions develop RB3LYP-UB3LYP instability with increasing oligomer length, and unrestricted wave functions become lower in energy. A similar trend was observed in bare oligothiophene dications. ${ }^{21}$ The spin-restricted wave function is stable for the lowest energy isomer of short salts (e.g., $\left.8 \mathrm{~T}^{2+}\left(\mathrm{Cl}_{3}{ }^{-2}(4)\right)_{2}\right)$ and it can be considered as a pure bipolaron located at the center of the chain. For the medium-sized salts (e.g., $12 \mathrm{~T}^{2+}\left(\mathrm{Cl}_{3}{ }^{-}\right)_{2}$ the restricted wave function is unstable for the most stable structure $\left(12 \mathrm{~T}^{2+}\left(\mathrm{Cl}_{3}{ }^{-}(5)\right)_{2}\right)$ (Table 1). ${ }^{43}$ At the same time, the energy difference between the most stable structure $\left(12 \mathrm{~T}^{2+}\left(\mathrm{Cl}_{3}{ }^{-}(5)\right)_{2}\right)$ and the lowest energy structure at RB3LYP $\left(12 \mathrm{~T}^{2+}\left(\mathrm{Cl}_{3}{ }^{-}(6)\right)_{2}\right)$ 
is only $0.4 \mathrm{kcal} / \mathrm{mol}$. Thus, the polaron pair state makes a contribution to the electronic structure of medium-sized oligothiophenes in the presence of a dopant (at the hybrid DFT B3LYP level of theory) and the electronic state of $12 \mathrm{~T}^{2+}\left(\mathrm{Cl}_{3}-(5)\right)_{2}$ can be characterized as a mixture of bipolaron and polaron-pair states. For the long salts (e.g., $\left.20 \mathrm{~T}^{2+}\left(\mathrm{Cl}_{3}{ }^{-}\right)_{2}\right)$ the most stable structure at spin-unrestricted state $\left(20 \mathrm{~T}^{2+}\left(\mathrm{Cl}_{3}{ }^{-}(6)\right)_{2}\right)$ is significantly more stable and the polaron pair state is the dominant state. These observations reveal that the contribution of the polaron-pair state increases with conjugated chain length and becomes the dominant state in the presence of dopant for systems of sufficient length (20T and longer). This trend is consistent with our recent study on oligothiophene dications where we found that the energy difference between restricted and unrestricted wave functions increases with dication chain length, for example $\Delta E_{\mathrm{RB} 3 \mathrm{LYP}}$ UB3LYP is $1.6 \mathrm{kcal} / \mathrm{mol}$ for $8 \mathrm{~T}^{2+}$ and $4.9 \mathrm{kcal} / \mathrm{mol}$ for $20 \mathrm{~T}^{2+} .21$ However, in oligothiophene divalent salts a chain length of eight rings is still not sufficiently long for the bipolaron to become intrinsically unstable, which is in contrast to the situation with bare oligothiophene dications ${ }^{18,21}$ where the bipolaron becomes intrinsically unstable at a chain length of six rings. Because of flat potential energy surfaces, there are a number of isomers of $20 \mathrm{~T}^{2+}\left(\mathrm{Cl}_{3}{ }^{-}\right)_{2}$ with very similar energies (Table 1$)$. The isomers with counterions positioned next to rings $m=4,5$, and 7-9 in the spin-unrestricted states differ in energy from the most stable isomer by less than $1 \mathrm{kcal} / \mathrm{mol}$ (Table 1 ). This indicates that dopant ions can change their location between positions 4-9 nearly randomly.

If counterions are removed from oligothiophene divalent salts to form bare oligothiophene dications, the reorganization energies released during geometry relaxation are very small, 2.8, 2.7 , and $1.6 \mathrm{kcal} / \mathrm{mol}$ for $8 \mathrm{~T}^{2+}\left(\mathrm{Cl}_{3}{ }^{-}(4)\right)_{2}, 12 \mathrm{~T}^{2+}\left(\mathrm{Cl}_{3}{ }^{-}(5)\right)_{2}$, and $20 \mathrm{~T}^{2+}\left(\mathrm{Cl}_{3}{ }^{-}(6)\right)_{2}$, respectively. The small relaxation energies are in agreement with our previous finding for doped oligothiophene cations. ${ }^{27}$ The decrease in reorganization energy with increasing chain length parallels the observed decrease in energy for positive charge hoping in long oligothiophenes. ${ }^{50,51}$

In general, for the calculated minimal energy structures of oligothiophene divalent salts, separation of the two counterions from each other increases with increasing chain length. The BLA patterns (Figure 3a) of short salts indicate that there is no separation between the two positive sites on the chain, and hence the pattern suggests a bipolaron state for all $8 \mathrm{~T}^{2+}\left(\mathrm{Cl}_{3}{ }^{-}\right)_{2}$ isomers. A similar trend is observed for the charge distribution (see Figure $6 a$ ). The BLA pattern for the triplet state (Figure 7a) of $8 \mathrm{~T}^{2+}\left(\mathrm{Cl}_{3}{ }^{-}(4)\right)_{2}$ bears little similarity to the corresponding singlet state, and this suggests that the contribution of the polaron pair to $8 \mathrm{~T}^{2+}\left(\mathrm{Cl}_{3}{ }^{-}(4)\right)_{2}$ is low. The charge distribution in the triplet state of $8 \mathrm{~T}^{2+}\left(\mathrm{Cl}_{3}{ }^{-}(4)\right)_{2}$ demonstrates slightly more charge delocalization than for the corresponding singlet state (Figure 8a). We also note that strong delocalization, as observed in bare dications $^{21}$ is not observed in divalent salts, although some charge delocalization over the 5-8 rings located around the position of the dopant is observed.

The BLA pattern of $12 \mathrm{~T}^{2+}\left(\mathrm{Cl}_{3}{ }^{-}(5)\right)_{2}$ suggests a mainly bipolaronic electronic structure and contribution from the polaron-pair state is small, although the restricted wave function is unstable for this isomer (see Table 1). This is in contrast to bare $12 \mathrm{~T}^{2+}$, where the BLA pattern suggests a polaron-pair state (Figure 3b). Thus, introduction of a dopant shifts the bipolaronpolaron-pair equilibrium toward the bipolaron state. Isomers with separated counterions, such as $12 \mathrm{~T}^{2+}\left(\mathrm{Cl}_{3}{ }^{-}(2)\right)_{2}$, clearly indicate dissociation of a bipolaron into a polaron-pair (in agreement
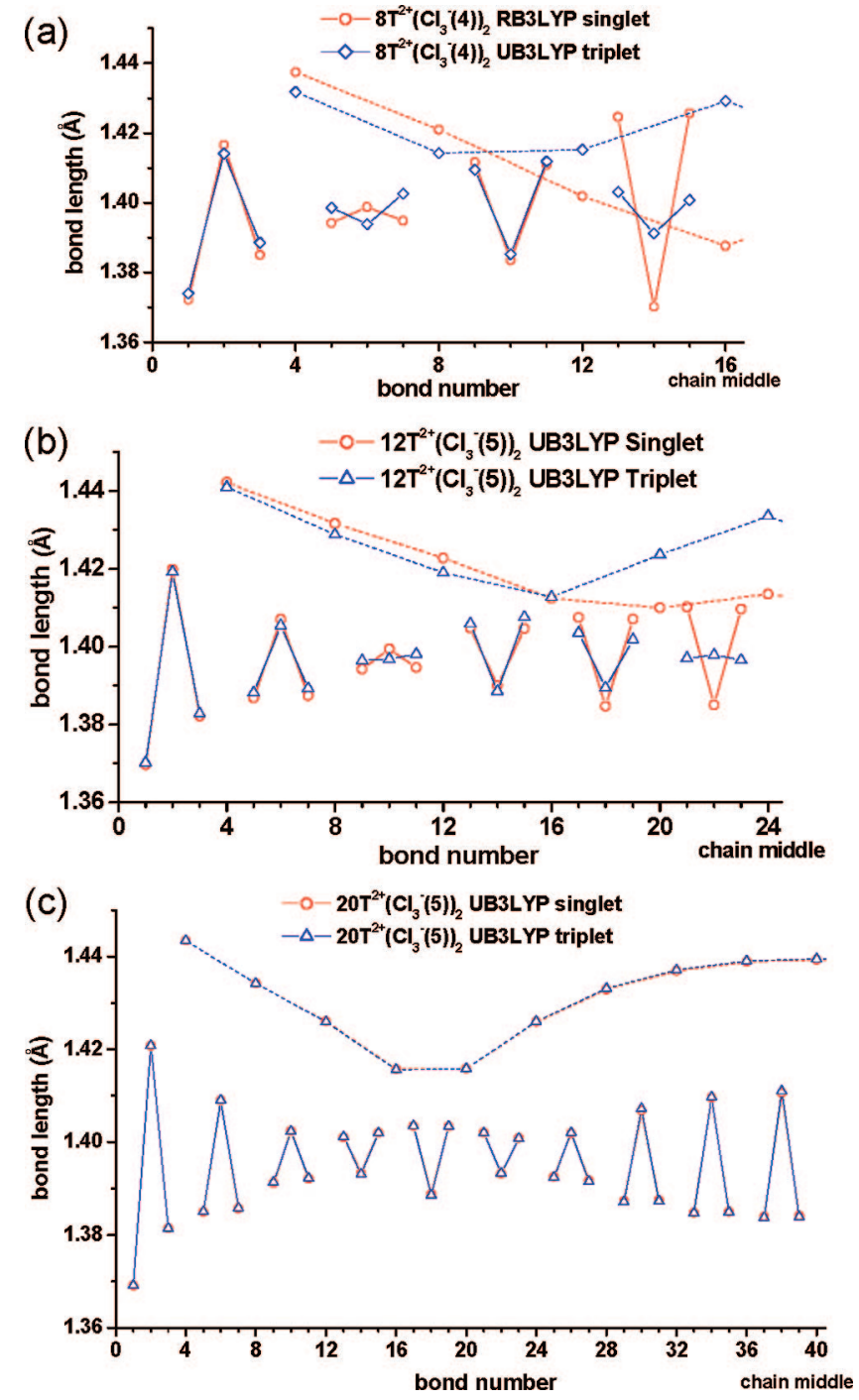

Figure 7. Comparison of BLA patterns for singlet and triplet states of oligothiophene divalent salts (a) $8 \mathrm{~T}^{2+}\left(\mathrm{Cl}_{3}{ }^{-}(4)\right)_{2}$, (b) $12 \mathrm{~T}^{2+}\left(\mathrm{Cl}_{3}{ }^{-}(5)\right)_{2}$, and (c) $20 \mathrm{~T}^{2+}\left(\mathrm{Cl}_{3}{ }^{-}(5)\right)_{2}$. See caption to Figure 8 .

with Figure $3 \mathrm{~b}$ above), however, this isomer is not the minimal energy structure. The geometry obtained for the triplet state (Figure $7 \mathrm{~b}$ ) of the $12 \mathrm{~T}^{2+}\left(\mathrm{Cl}_{3}{ }^{-}(5)\right)_{2}$ isomer is somewhat similar to the geometry of the singlet state; however, the central rings are slightly more aromatic in the triplet state. For $12 \mathrm{~T}^{2+}\left(\mathrm{Cl}_{3}{ }^{-}(5)\right)_{2}$, the charge distribution patterns obtained for the singlet and triplet states are already quite similar (Figure $8 \mathrm{~b}$ ), also suggesting some contribution from the polaron-pair state. This is consistent with the decrease in $\Delta E_{\mathrm{T}-\mathrm{S}}$ for $12 \mathrm{~T}^{2+}\left(\mathrm{Cl}_{3}{ }^{-}(5)\right)_{2}$ relative to $8 \mathrm{~T}^{2+}\left(\mathrm{Cl}_{3}{ }^{-}(4)\right)_{2}$ (from 8.4 to $3.1 \mathrm{kcal} /$ mol, Table 1) and suggests some possible contribution from the polaron pair states to the electronic structure of singlet $12 \mathrm{~T}^{2+}\left(\mathrm{Cl}_{3}{ }^{-}(5)\right)_{2}$. The BLA pattern of $20 \mathrm{~T}^{2+}\left(\mathrm{Cl}_{3}{ }^{-}(5)\right)_{2}$ indicates a clear separation of the two polarons. This is consistent with the significant difference $(7.9 \mathrm{kcal} / \mathrm{mol})$ between RB3LYP and UB3LYP energies for $20 \mathrm{~T}^{2+}\left(\mathrm{Cl}_{3}{ }^{-}(5)\right)_{2}$. Interestingly, the BLA patterns of the singlet and triplet states of $20 \mathrm{~T}^{2+}\left(\mathrm{Cl}_{3}{ }^{-}(5)\right)_{2}$ are practically identical (Figure 7c), which together with their energetic degeneracy (see Table 1) suggests two well defined polarons (one on each side of the chain) without significant contribution from the bipolaron state. Mulliken charge distributions in both singlet and triplet states of $20 \mathrm{~T}^{2+}\left(\mathrm{Cl}_{3}{ }^{-}(5)\right)_{2}$ are also practically identical (Figure 8c), which strongly points to their similar electronic nature, both being polaron-pairs. 

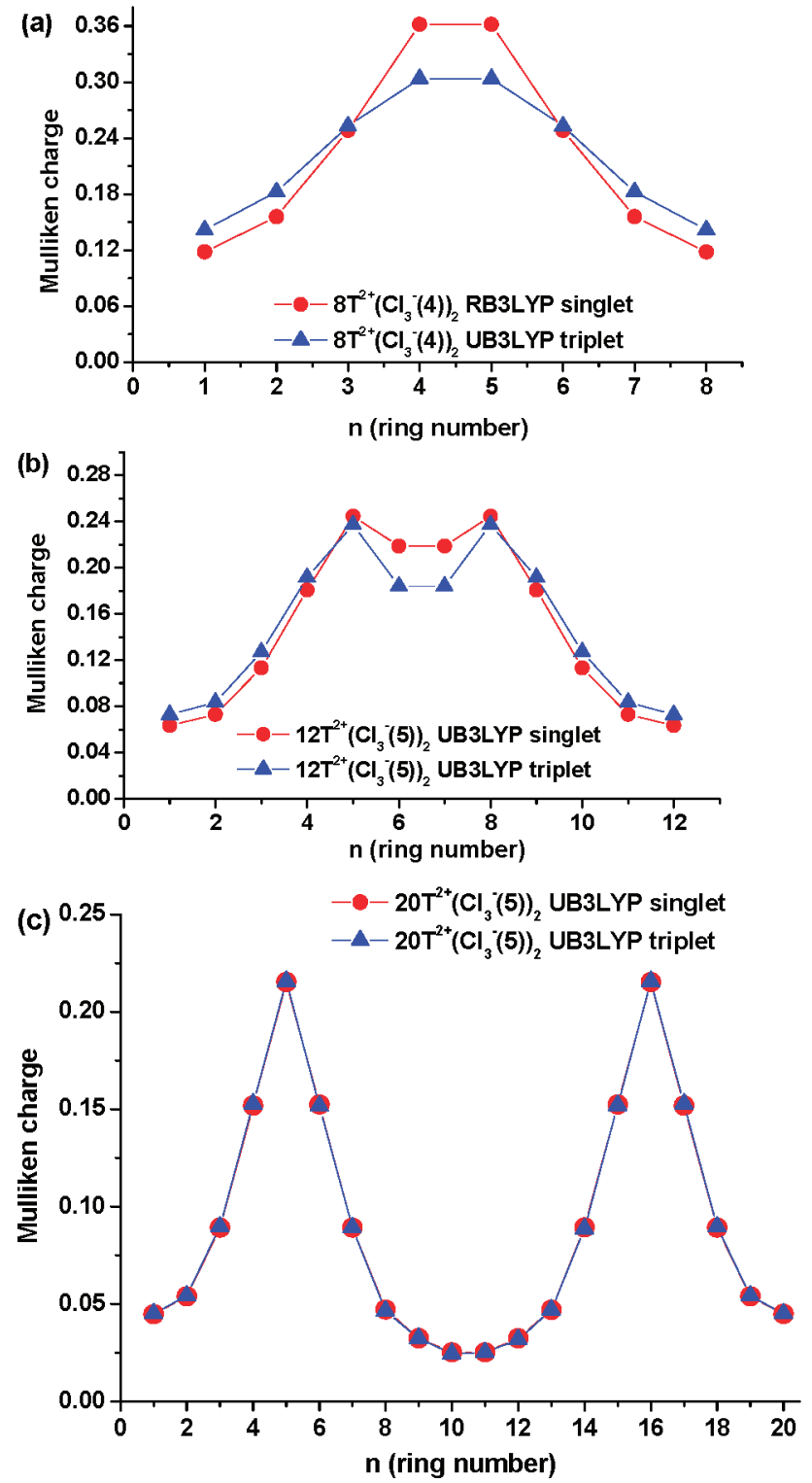

Figure 8. Mulliken charge distribution summarized for thiophene rings (at B3LYP/6-31G(d)) for singlet and triplet states of (a) $8 \mathrm{~T}^{2+}\left(\mathrm{Cl}_{3}{ }^{-}(4)\right)_{2}$, (b) $12 \mathrm{~T}^{2+}\left(\mathrm{Cl}_{3}^{-}(5)\right)_{2}$, and (c) $20 \mathrm{~T}^{2+}\left(\mathrm{Cl}_{3}^{-}(5)\right)_{2}$.

On the basis of geometric distortions of the oligothiophene backbone, it is clear that the bipolaron becomes unstable toward dissociation into a polaron-pair even in the presence of dopant if the oligomer chain is sufficiently long. Compared to our previous studies of bare oligothiophene dications ${ }^{21}$ and polycations $^{22}$ and to studies of the dications of several other short conjugated oligomer ${ }^{20}$ in the absence of counterions, the chain length at which bipolarons become unstable is larger in the presence of counterions. This is consistent with defect sizes decreasing in the presence of counterions. On the basis of analysis of charge distribution pattern, it is clear that the bipolaron will be unstable and will dissociate into two polarons at some oligothiophene length. ${ }^{52}$ Provided the chain is of sufficient length, dopant ions avoid being close to the chain ends and each other. Beyond these constraints, the dopant position is flexible over the oligothiophene chain.

In our previous paper, ${ }^{21}$ we have shown that the singlet state of short and medium length bare oligothiophene dications is more stable than the triplet state and that both states become practically energetically degenerate at the long chain limit. Degeneracy between singlet and triplet states is characteristic of polaron pairs. The presence of counterions increases the singlet-triplet energy difference which points to a more bipolaronic nature for doped salts compared to bare dications. Values of $\Delta E_{\mathrm{T}-\mathrm{S}}$ for bare oligothiophene dications are significantly smaller. Thus, the singlet state of $8 \mathrm{~T}^{2+}$ and $12 \mathrm{~T}^{2+}$ is 2.7 and $0.7 \mathrm{kcal} / \mathrm{mol}$, respectively, lower in energy than the triplet state (while the singlet state of $8 \mathrm{~T}^{2+}\left(\mathrm{Cl}_{3}-(4)\right)_{2}$ and $12 \mathrm{~T}^{2+}\left(\mathrm{Cl}_{3}{ }^{-}(5)\right)_{2}$ is 8.4 and $3.1 \mathrm{kcal} / \mathrm{mol}$, respectively, lower in energy than the triplet state); however for $20 \mathrm{~T}^{2+}$ and $20 \mathrm{~T}^{2+}\left(\mathrm{Cl}_{3}{ }^{-}(6)\right)_{2}$, the two states are practically degenerate with an energy difference of less than $0.1 \mathrm{kcal} / \mathrm{mol}^{2}{ }^{21}$ The fact that singlet and triplet states become energetically degenerate for doped oligothiophene salts of sufficient length also suggests that long oligothiophenes prefer the polaron-pair state even in the presence of a dopant.

The small synproportionation energies that were found for divalent salts (eq 1 and Table 1) should be compared to those of bare oligothiophene dications (eq 5) that release $53.4 \mathrm{kcal} /$ $\mathrm{mol}$ for $8 \mathrm{~T}^{2+}$ and $24.0 \mathrm{kcal} / \mathrm{mol}$ for $20 \mathrm{~T}^{2+}$. Thus, the presence of counterions reduces synproportiontion energies by at least an order of magnitude, which reflects weaker interactions between the two positive charges in doped oligothiophenes compared to bare oligothiophene dications. In the case of $20 \mathrm{~T}^{2+}\left(\mathrm{Cl}_{3}{ }^{-}\right)_{2}$, the charge separation reaction is practically thermoneutral, indicating that the two charges are well separated, which is in agreement with the BLA pattern and charge distribution, and that its electronic structure can be described as a practically separated polaron pair. ${ }^{53}$ The stability of divalent salts relative to two oligothiophene monovalent salts of half the chain length (eq 2) revealed that short (e.g., $\left.8 \mathrm{~T}^{2+}\left(\mathrm{Cl}_{3}{ }^{-}\right)_{2}\right)$ divalent salt is more stable, which might correspond to the bipolaron stabilization energy. For long salts, this equation is thermoneutral, which in turn points to the absence of any bipolaron stabilization energy if the oligothiophene chain is sufficiently long.

Counterions substantially reduce the disproportion energies of monocations into dications and neutral chains, but the process remains endothermic in all cases investigated. Thus, the present study suggests that at low doping levels polarons are formed in oligothiophenes of any chain length. Because pairing of polarons into bipolarons is only slightly endothermic in the presence of counterions, bipolarons may form at high-doping levels. This is caused by space limitation and not by a bipolaron binding energy. ${ }^{54}$

\section{Conclusions}

Long-doped oligothiophenes (up to 20 thiophene units) were modeled for the first time by oligothiophene divalent salts comprised of oligothiophenes and counterions in which the counterion is not covalently bound to the oligothiophene chain. Introducing counterions to the system is reflected by geometry distortions and localization of charges on the rings adjacent to the counterion position, that is, charges are more localized compared to bare oligothiophene dications, although some charge delocalization is always observed. This study (in combination with our previous study) ${ }^{21}$ therefore succeeds in spanning the electronic configuration range within which real doped poly- and oligothiophenes must fall, by describing the configurations of both bare dications and gas phase oligothiophene salts.

On the basis of various criteria (at the B3LYP/6-31G(d) level of theory), such as the relative energies of the open-shell singlet, closed-shell singlet and triplet states, bond length alternation patterns, and charge distribution analysis, we conclude that in 
short oligothiophene divalent salts (such as $\left.8 \mathrm{~T}^{2+}\left(\mathrm{Cl}_{3}^{-}\right)_{2}\right)$ the major contribution to electronic structure comes from the bipolaron state. For medium-sized oligothiophene divalent salts (such as $12 \mathrm{~T}^{2+}\left(\mathrm{Cl}_{3}{ }^{-}\right)_{2}$ ), some contribution from the polaron pair state is observed, and in the long oligothiophene divalent salts (such as $20 \mathrm{~T}^{2+}\left(\mathrm{Cl}_{3}{ }^{-}\right)_{2}$ ), the polaron pair state is dominant. We have clearly seen the transition from bipolaron (in $\left.8 \mathrm{~T}^{2+}\left(\mathrm{Cl}_{3}{ }^{-}\right)_{2}\right)$ to polaron pair (in $20 \mathrm{~T}^{2+}\left(\mathrm{Cl}_{3}^{-}\right)_{2}$ ) ground states in doped oligothiophenes, and we can conclude (in contrast to early theoretical studies ${ }^{10,15}$ ) that bipolarons are intrinsically unstable with respect to dissociation into polaron pair regardless of the presence of counterions.

Compared to previous studies of bare oligothiophene dications, the chain length at which bipolarons become unstable is larger in the presence of counterions. At shorter chain lengths that induce the polarons to interact, all oligomer dications investigated here have singlet ground states. At the long chain limit, singlet and triplet oligothiophene salts are energetically degenerate and have almost identical geometries.

Acknowledgment. We thank the Israel Science Foundation, the Helen and Martin Kimmel Center for Molecular Design, TUBITAK (TBAG 2461), and Bilkent University for financial support. M.B. is the incumbent of the Recanati career development chair, a member ad personam of the Lise Meitner-Minerva Center for Computational Quantum Chemistry and acknowledges DuPont for a Young Professor Award.

Supporting Information Available: Tables of absolute energies; the Cartesian coordinates of the optimized geometries of the divalent and monovalent salt isomers; results of calculations of monovalent salts; graphs of isomers relative energies and $\Delta E_{\mathrm{T}-\mathrm{S}}$; graphs of charge distribution and bond length alternation; density of states patterns and molecular orbital images. This material is available free of charge via the Internet at http://pubs.acs.org.

\section{References and Notes}

(1) (a) Electronic Materials: The Oligomer Approach; Müllen, K. Wegner, G., Eds.; Wiley-VCH: Weinheim, Germany, 1998. (b) Handbook of Oligo- and Polythiophenes; Fichou, D., Ed.; Wiley-VCH: Weinheim, Germany, 1999. (c) Handbook of Conducting Polymers, 2nd ed.; Skotheim, T. A., Elsenbaumer, R. L., Reynolds, J. R., Eds.; Marcel Dekker: New York, 1998. (d) Conjugated Polymers: The Novel Science and Technology of Highly Conducting and Nonlinear Optically Active Materials; Brédas, J. L., Silbey, R., Eds.; Kluwer Academic Publishing: Dordrecht, The Netherlands, 1991. (e) Handbook of Organic Conductive Molecules and Polymers; Nalwa, H. S., Ed.; John Wiley \& Sons: New York, 1997; Vols. 1-4. (f) Handbook of Conducting Polymers. Conjugated Polymers: processing and applications, 3nd ed.; Skotheim, T. A., Reynolds, J. R., Eds.; CRC Press: Boca Raton, FL, 2007. (g) Tour, J. M. Chem. Rev. 1996, 96, 537. (h) Roncali, J. Chem. Rev. 1997, 97, 173. (i) Groenendaal, L. B.; Jonas, F.; Freitag, D.; Pielartzik, H.; Reynolds, J. R. Adv. Mater. 2000, 12, 481. (j) Perepichka, I. F.; Perepichka, D. F.; Meng, H.; Wudl, F. Adv. Mater. 2005, 17, 2281.

(2) (a) Horowitz, G.; Peng, X.; Fichou, D.; Garnier, F. J. Appl. Phys. 1990, 67, 528. (b) Paloheimo, J.; Kuivalainen, P.; Stubb, H.; Vuorimaa E.; Yli-Lahti, P. Appl. Phys. Lett. 1990, 56, 1157. (c) Garnier, F.; Hajlaoui, R.; Yassar, A.; Srivastava, P. Science 1994, 265, 1684. (d) Dodabalapur, A.; Katz, H. E.; Torsi, L.; Haddon, R. C. Science 1995, 269, 1560. (e) Dimitrakopoulos, C. D.; Malenfant, P. R. L. Adv. Mater. 2002, 14, 99. (f) Horowitz, G. Adv. Mater. 1998, 10, 365. (g) Katz, H. E. J. Mater. Chem. 1997, 7, 369. (h) Halik, M.; Klauk, H.; Zschieschang, U.; Schmid, G.; Ponomarenko, S.; Kirchmeyer, S.; Weber, W. Adv. Mater. 2003, 15, 917.

(3) Tessler, N.; Veres, J.; Globerman, O.; Rappaport, N.; Preezant, Y.; Roichman, Y.; Solomesch, O.; Tal, S.; Gershman, E.; Adler, M.; Zolotarev, V.; Gorelik, V.; Eichen, Y. In Handbook of Conducting Polymers. Conjugated Polymers: processing and applications, 3nd ed.; Skotheim, T. A., Reynolds, J. R., Eds.; CRC Press: Boca Raton, FL, 2007; Chapter 7, p 7-1.

(4) (a) Geiger, F.; Stoldt, M.; Schweizer, H.; Bäuerle, P.; Umbach, E. Adv. Mater. 1993, 5, 922. (b) Mitschke, U.; Bäuerle, P. J. Mater. Chem. 2000, 10, 1471. (c) Barbarella, G.; Favaretto, L.; Sotgiu, G.; Zambianchi,
P.; Bongini, A.; Arbizzani, C.; Mastragostino, M.; Anni, M.; Gigli, G.; Cingolani, R. J. Am. Chem. Soc. 2000, 122, 11971. (d) Barbarella, G.; Favaretto, L.; Sotgiu, G.; Antolini, L.; Gigli, G.; Cingolani, R.; Bongini, A. Chem. Mater. 2001, 13, 4112.

(5) Robinson, N. D.; Berggren, M. In Handbook of Conducting Polymers. Conjugated Polymers: processing and applications, 3nd ed.; Skotheim, T. A., Reynolds, J. R., Eds.; CRC Press: Boca Raton, FL, 2007; Chapter 4, p 4-1.

(6) (a) Brabec, C. J.; Sariciftci, N. S.; Hummelen, J. C. Adv. Funct. Mater. 2001, 11, 15. (b) Hoppe, H.; Sariciftci, N. S. J. Mater. Res. 2004, 19, 1924.

(7) Mozer, A. J.; Sariciftci, N. S. In Handbook of Conducting Polymers. Conjugated Polymers: processing and applications, 3nd ed.; Skotheim, T. A., Reynolds, J. R., Eds.; CRC Press: Boca Raton, FL, 2007; Chapter $10, \mathrm{p} 10-1$.

(8) Dyer, A. L.; Reynolds, J. R. In Handbook of Conducting Polymers. Conjugated Polymers: theory, synthesis, properties, and characterization, 3nd ed.; Skotheim, T. A., Reynolds, J. R., Eds.; CRC Press: Boca Raton, FL, 2007; Chapter 20, p 20-1.

(9) Patil, A. O.; Heeger, A. J.; Wudl, F. Chem. Rev. 1988, 88, 183. (10) (a) Heeger, A. J.; Kivelson, S.; Schrieffer, J. R.; Su, W. P. Rev. Mod. Phys. 1988, 60, 781. (b) Brédas, J. L.; Street, G. B. Acc. Chem. Res. 1985, 18, 309. (c) Miller, L. L.; Mann, K. R. Acc. Chem. Res. 1996, 29 , 417. (d) Salaneck, W. R.; Friend, R. H.; Brédas, J. L. Phys. Rep. 1999, 319,231

(11) Furukawa, Y. J. Phys. Chem. 1996, 100, 15644.

(12) Brédas, J. L.; Beljonne, D.; Coropceanu, V.; Cornil, J. Chem. Rev. 2004, 104, 4971.

(13) (a) Kaneto, K.; Hayashi, S.; Ura, S.; Yoshino, K. J. Phys. Soc. Jpn. 1985, 54, 1146. (b) Chen, J.; Heeger, A. J.; Wudl, F. Solid State Commun. 1986, 58, 251. (c) Colaneri, N.; Nowak, M.; Spiegel, D.; Hotta, S.; Heeger, A. J. Phys. Rev. B 1987, 36, 7964. (d) Fichou, D.; Horowitz, G.; Xu, B.; Gamier, F. Synth. Met. 1990, 39, 243. (e) Harima, Y.; Eguchi, T.; Yamashita, K.; Kojima, K.; Shiotani, M. Synth. Met. 1999, 105, 121.

(14) van Haare, J. A. E. H.; Havinga, E. E.; van Dongen, J. L. J.; Janssen, R. A. J.; Cornil, J.; Brédas, J. L. Chem. Eur. J. 1998, 4, 1509.

(15) (a) Brédas, J. L.; Thémans, B.; André, J. M.; Chance, R. R.; Silby, R. Synth. Met. 1984, 9, 265. (b) Brédas, J. L.; Thémans, B.; Fripiat, J. G.; André, J. M.; Chance, R. R. Phys. Rev. B 1984, 29, 6761. (c) Brédas, J. L.; Street, G. B.; Acc. Chem. Res. 1985, 18, 309. (d) Brédas, J. L.; Wudl, F.; Heeger, A. J. Solid State Commun. 1987, 63, 577. (e) Bertho, D.; Jouanin, C. Phys. Rev. B 1987, 35, 626. (f) Silva, G. M. E. Phys. Rev. B 2000, 61, 10777.

(16) (a) Tol, A. J. W. Synth. Met. 1995, 74, 95. (b) Tol, A. J. W. Chem. Phys. 1996, 208, 73.

(17) Brocks, G. Synth. Met. 1999, 102, 914.

(18) Gao, Y.; Liu, C.-G.; Jiang, Y.-S. J. Phys. Chem. A 2002, 106, 5380.

(19) Geskin, V. M.; Brédas, J. L. ChemPhysChem 2003, 4, 498.

(20) Casanovas, J.; Alemán, C. J. Phys. Chem. C 2007, 111, 4823.

(21) Zade, S. S.; Bendikov, M. J. Phys. Chem. B 2006, 110, 15839.

(22) Zade, S. S.; Bendikov, M. J. Phys. Chem. C 2007, 111, 10662.

(23) Chiu, W. W.; Travaš-Sejdiæ, J.; Cooney, R. P.; Bowmaker, G. A. Synth. Met. 2005, 155, 80.

(24) (a) Irle, S.; Lischka, H.; Eichkom, K.; Ahlrichs, R. Chem. Phys. Lett. 1996, 257, 592. (b) Irle, S.; Lischka, H. J. Chem. Phys. 1997, 107, 3021.

(25) Champagne, B.; Spassova, M. Phys. Chem. Chem. Phys. 2004, 6, 3167.

(26) Sing-Miller, N. E.; Scherlis, D. A.; Marzari, N. J. Phys. Chem. B 2006, 110, 24822.

(27) Salzner, U. J. Chem. Theory Comput. 2007, 3, 1143.

(28) Frisch, M. J.; Trucks, G. W.; Schlegel, H. B.; Scuseria, G. E.; Robb, M. A.; Cheeseman, J. R.; Montgomery, J. A., Jr.; Vreven, T.; Kudin, K. N.; Burant, J. C.; Millam, J. M.; Iyengar, S. S.; Tomasi, J.; Barone, V.; Mennucci, B.; Cossi, M.; Scalmani, G.; Rega, N.; Petersson, G. A.; Nakatsuji, H.; Hada, M.; Ehara, M.; Toyota, K.; Fukuda, R.; Hasegawa, J.; Ishida, M.; Nakajima, T.; Honda, Y.; Kitao, O.; Nakai, H.; Klene, M.; Li, X.; Knox, J. E.; Hratchian, H. P.; Cross, J. B.; Adamo, C.; Jaramillo, J.; Gomperts, R.; Stratmann, R. E.; Yazyev, O.; Austin, A. J.; Cammi, R.; Pomelli, C.; Ochterski, J. W.; Ayala, P. Y.; Morokuma, K.; Voth, G. A.; Salvador, P.; Dannenberg, J. J.; Zakrzewski, V. G.; Dapprich, S.; Daniels, A. D.; Strain, M. C.; Farkas, O.; Malick, D. K.; Rabuck, A. D.; Raghavachari, K.; Foresman, J. B.; Ortiz, J. V.; Cui, Q.; Baboul, A. G.; Clifford, S.; Cioslowski, J.; Stefanov, B. B.; Liu, G.; Liashenko, A.; Piskorz, P.; Komaromi, I.; Martin, R. L.; Fox, D. J.; Keith, T.; Al-Laham, M. A.; Peng, C. Y.; Nanayakkara, A.; Challacombe, M.; Gill, P. M. W.; Johnson, B.; Chen, W.; Wong, M. W.; Gonzalez, C.; Pople, J. A. Gaussian 03, Revision C.02; Gaussian, Inc.: Wallingford, CT, 2004.

(29) (a) Parr, R. G.; Yang, W. Density-functional theory of atoms and molecules; Oxford University Press: New York, 1989. (b) Koch, W.; Holthausen, M. C. A Chemist's guide to density functional theory; WileyVCH: New York, 2000. (c) Lee, C.; Yang, W.; Parr, R. G. Phys. Rev. B 1988, 37, 785. (d) Becke, A. D. J. Chem. Phys. 1993, 98, 5648. 
(30) Open-shell singlets calculated using broken-symmetry DFT methods are not pure spin states, which is reflected by their $S^{2}$ values that differ from 0 (pure singlet) or 2 (pure triplet).

(31) Gruhn, N. E.; da Silva Filho, D. A.; Bill, T. G.; Malagoli, M.; Coropceanu, V.; Kahn, A.; Brédas, J. L. J. Am. Chem. Soc. 2002, 124 7918.

(32) Correlated ab initio methods can not be used in this study because they can be applied only to relatively small molecules, and polaronbipolaron equilibrium is not observed for small systems ( The DFT and HF levels of theory are the only practical options available for studying doped oligothiophenes of sufficient length to enable the transition from bipolaron to polaron-pair to be observed. The HF level is inapplicable to the study of oligothiophene dications (as well as other charged conjugated systems) due to the huge spin contamination obtained if a pure $\mathrm{HF}$ wave function is used. ${ }^{19}$

(33) Cai, Z.-L.; Sendt, K.; Reimers, J. R. J. Chem. Phys. 2002, 117, 5543.

(34) Bally, T.; Hrovat, D. A.; Thatcher Borden, W. Phys. Chem. Chem. Phys. 2000, 2, 3363

(35) Salzner, U. J. Chem. Theor. Comput. 2007, 3, 219.

(36) Kertesz, M.; Choi, C. H.; Yang, S. Chem. Rev. 2005, 105, 3448

(37) Zade, S. S.; Bendikov, M. Org. Lett. 2006, 8, 5243.

(38) We have tested the applicability of the broken-symmetry B3LYP/ 6-31G(d) level to appropriately describe oligothiophene dications by comparing it to CASSCF results. ${ }^{21}$ Both methods agreed qualitatively on the relative energies of singlet and triplet oligothiophene dications and on the electronic structure (bipolaron versus polaron pair) of the dications.

(39) Davidson, E. R.; Clark, A. E. Int. J. Quantum Chem. 2005, 103, 1

(40) Kraka, E.; Cremer, D. J. Comput. Chem. 2001, 22, 216.

(41) The position of counterion in-plane with the oligothiophene chains was found to be true minima according to frequency analysis of $8 \mathrm{~T}^{+} \mathrm{Cl}_{3}$ (see Supporting Information).

(42) The calculation of oligothiophene monovalent salts $\mathrm{nT}^{+} \mathrm{Cl}_{3}{ }^{-}$and $\mathrm{nT}^{+} \mathrm{Br}_{3}{ }^{-}$(see Supporting Information) revealed that counterions not covalently bounded to oligomer backbone as evident by shortest $\mathrm{H} \cdot \cdots \mathrm{X}$ distances of 2.59-2.60 $\AA$ for $\mathrm{Cl}_{3}{ }^{-}$and 2.71-2.85 $\AA$ for $\mathrm{Br}_{3}{ }^{-}$.

(43) Wave function instability can be expected for low HOMO-LUMO gap systems as the number of conjugated double bonds increases. Wave function instability has been observed previously for bare oligothiophene dications. ${ }^{18,21}$ Similarly, RHF-UHF wave function instability has been predicted for other types of long conjugated systems, such as oligoacenes starting from hexacene at B3LYP/6-31G(d); see Bendikov, M.; Duong, H. M.; Starkey, K.; Houk, K. N.; Carter, E. A.; Wudl, F. J. Am. Chem. Soc. 2004, 126, 7416.

(44) When the $C_{1}-C_{2}$ and $C_{3}-C_{4}$ bonds of a thiophene ring (Scheme 1) are shorter than the $C_{2}-C_{3}$ bond (so producing a $\Lambda$-shaped pattern in the sets of three linked data points shown in Figure 3), the ring is considered aromatic. By contrast, when the $\mathrm{C}_{1}-\mathrm{C}_{2}$ and $\mathrm{C}_{3}-\mathrm{C}_{4}$ bonds of a thiophene ring are longer than the $\mathrm{C}_{2}-\mathrm{C}_{3}$ bond (thus, producing a $\mathrm{V}$-shaped pattern in the sets of three linked data points shown the Figure 3), the ring is considered quinoid (Scheme 1).

(45) BLA patterns for other isomers are available in Supporting Information.

(46) The calculated density of states of the 20T, 20T ${ }^{2+}, 20 \mathrm{~T}^{2+}\left(\mathrm{Cl}_{3}{ }^{-}\right.$ (5) $)_{2}$ and $20 \mathrm{~T}^{2+}\left(\mathrm{Cl}_{3}-(10)\right)_{2}$ are presented in Supporting Information.

(47) The fact that $\alpha$ and $\beta$ orbitals are mirror images of each other resembles frontier orbital picture of long oligoacenes that correspond to disjoint diradicals; see ref 43 and references therein.

(48) Roncali, J. Chem. Rev. 1992, 92, 711.

(49) (a) Kobayashi, M.; Chen, J.; Chung, T. C.; Moraes, F.; Heeger, A. J.; Wudl, F. Synth. Met. 1984, 9, 77. (b) Oztemiz, S.; Beaucage, G.; Ceylan, O.; Mark, H. B. J. Solid State Electrochem. 2004, 8, 928.

(50) Zade, S. S.; Bendikov, M. Chem. Eur. J. 2008, 14, in press (DOI: 10.1002/chem.200701182).

(51) The shortest oligothiophene length at which a bipolaron will become unstable and dissociate into two polarons (a polaron-pair) will depend (and might even strongly depend) on the level of theory used (see Theoretical Methods and the discussion about charge distribution). However, it is clear that the bipolaron will be unstable and will dissociate into two polarons at some oligothiophene length.

(52) We note that the charge distribution pattern in oligothiophene cation radicals is significantly dependent on the amount of $\mathrm{HF}$ exchange used. Localization of charges is proportional to the amount of HF exchange with charge distribution very localized at the HF level and practically completely delocalized at pure DFT levels (Zade, S. S.; Bendikov, M., unpublished. Similar conclusions were also drawn for oligo(phenylene vinylene) cation radicals; see Geskin, V. M.; Grozema, F. C.; Siebbeles, L. D. A.; Bejonne, D.; Brédas, J. L.; Cornil, J. J. Phys. Chem. B 2005, 109, 20237). Although presently it is unclear which theoretical method reproduces the charge distribution pattern correctly, it was also observed that for polyacetylene chains in the presence of counterions different levels of theory such as HF, MP2, and B3LYP provide similar bond length alternation and charge distribution patterns. ${ }^{25}$

(53) Unsurprisingly, extrapolation of eqs. 1 and 5 to infinite chain lengths results in an energetic degeneracy between the dication vs. two monocations and between divalent vs. two monovalent salts (Figure 5a).

(54) Preliminary estimation of solvent effect on the electronic structure of divalent oligothiophene salts was performed using PCM approximation. Inclusion of solvent favor the polaron pair state over the bipolaron state even more strongly compared to gas phase calculations of divalent oligothiophene salts (Zamoshchik, N.; Salzner, U.; Bendikov, M., unpublished).

JP7111582 